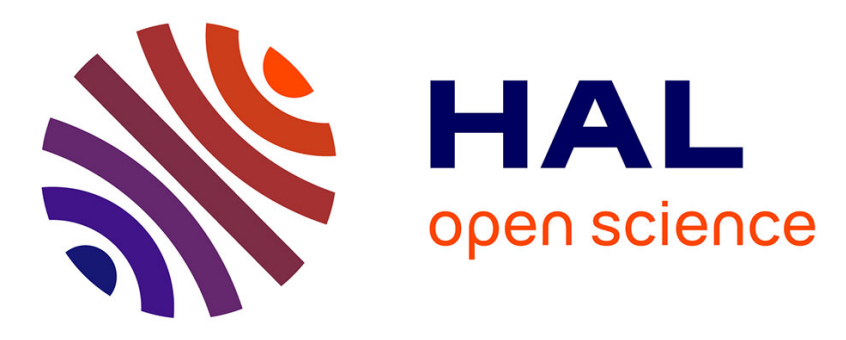

\title{
The von Karman Sodium experiment: Turbulent dynamical dynamos
}

\author{
Romain Monchaux, Michaël Berhanu, Sébastien Aumaître, Arnaud \\ Chiffaudel, François Daviaud, Bérengère Dubrulle, Florent Ravelet, Stéphan \\ Fauve, Nicolas Mordant, François Pétrélis, et al.
}

\section{To cite this version:}

Romain Monchaux, Michaël Berhanu, Sébastien Aumaître, Arnaud Chiffaudel, François Daviaud, et al.. The von Karman Sodium experiment: Turbulent dynamical dynamos. Physics of Fluids, 2010, 21 (3), pp.035108. 10.1063/1.3085724 . hal-00492266

\section{HAL Id: hal-00492266 https://hal.science/hal-00492266}

Submitted on 15 Jun 2010

HAL is a multi-disciplinary open access archive for the deposit and dissemination of scientific research documents, whether they are published or not. The documents may come from teaching and research institutions in France or abroad, or from public or private research centers.
L'archive ouverte pluridisciplinaire HAL, est destinée au dépôt et à la diffusion de documents scientifiques de niveau recherche, publiés ou non, émanant des établissements d'enseignement et de recherche français ou étrangers, des laboratoires publics ou privés. 


\title{
The von Kármán Sodium experiment: Turbulent dynamical dynamos
}

\author{
Romain Monchaux, ${ }^{1}$ Michael Berhanu, ${ }^{2}$ Sébastien Aumaître, ${ }^{1}$ Arnaud Chiffaudel, ${ }^{1}$ \\ François Daviaud, ${ }^{1}$ Bérengère Dubrulle, ${ }^{1}$ Florent Ravelet, ${ }^{1}$ Stephan Fauve, ${ }^{2}$ \\ Nicolas Mordant, ${ }^{2}$ François Pétrélis, ${ }^{2}$ Mickael Bourgoin, ${ }^{3}$ Philippe Odier, ${ }^{3}$ \\ Jean-François Pinton, ${ }^{3}$ Nicolas Plihon, ${ }^{3}$ and Romain Volk ${ }^{3}$ \\ ${ }^{1}$ CEA, IRAMIS, Service de Physique de l'Etat Condensé, CNRS URA 2464, CEA Saclay, \\ F-91191 Gif-sur-Yvette, France \\ ${ }^{2}$ Laboratoire de Physique Statistique, CNRS and École Normale Supérieure, 24 rue Lhomond, \\ F-75005 Paris, France \\ ${ }^{3}$ Laboratoire de Physique de l'École Normale Supérieure de Lyon, CNRS and Université de Lyon, \\ F-69364 Lyon, France
}

(Received 7 July 2008; accepted 19 January 2009; published online 30 March 2009;

publisher error corrected 2 April 2009)

\begin{abstract}
The von Kármán Sodium (VKS) experiment studies dynamo action in the flow generated inside a cylinder filled with liquid sodium by the rotation of coaxial impellers (the von Kármán geometry). We first report observations related to the self-generation of a stationary dynamo when the flow forcing is $\mathcal{R}_{\pi}$-symmetric, i.e., when the impellers rotate in opposite directions at equal angular velocities. The bifurcation is found to be supercritical with a neutral mode whose geometry is predominantly axisymmetric. We then report the different dynamical dynamo regimes observed when the flow forcing is not symmetric, including magnetic field reversals. We finally show that these dynamics display characteristic features of low dimensional dynamical systems despite the high degree of turbulence in the flow. (C) 2009 American Institute of Physics.
\end{abstract}

[DOI: $10.1063 / 1.3085724]$

\section{INTRODUCTION}

\section{A. Experimental dynamos}

Although it was almost a century ago that Larmor ${ }^{1}$ proposed that a magnetic field could be self-sustained by the motions of an electrically conducting fluid, ${ }^{2}$ the experimental demonstrations of this principle are quite recent. Using solid rotor motions, Lowes and Wilkinson realized the first (nearly homogeneous) experimental dynamo in $19633^{3}$ a subcritical bifurcation to a stationary magnetic field was generated and oscillations were later observed in an improved setup in which the angle between the rotors could be adjusted. ${ }^{4}$ In this experiment, the main induction source lies in the axial differential rotation between the rotor and the stationary soft iron. Observations of fluid dynamos, however, did not occur before 2000 with the Riga and Karlsruhe experiments. In Riga, the dynamo is generated by the screw motion of liquid sodium. The flow is a helicoidal jet confined within a bath of liquid sodium at rest. ${ }^{5}$ The induction processes are the differential rotation and the shear associated with the screw motion at the lateral boundary. The dynamo field has a helical geometry and is time periodic. The bifurcation threshold and geometry of the neutral mode trace back to Ponomarenko's analytical study ${ }^{6}$ and have been computed accurately with model velocity profiles ${ }^{7}$ or from Reynolds-averaged Navier-Stokes numerical simulations. ${ }^{8,9}$ The Karlsruhe dynamo $^{10}$ is based on scale separation, the flow being generated by motions of liquid sodium in an array of screw generators with like-sign helicity. The magnetic field at onset is stationary and its large scale component is transverse to the axis of the flow generators. The arrangement follows
Roberts' scheme ${ }^{11}$ and again the dynamo onset and magnetic field characteristics have been accurately predicted numerically. ${ }^{12-15}$

The approach adopted in the von Kármán Sodium (VKS) experiments builds up on the above results. In order to access various dynamical regimes above dynamo onset, ${ }^{16-18}$ it uses less constrained flow generators but keeps helicity and differential rotation as the main ingredients for the generation of magnetic field. ${ }^{19,20}$ It does not rely on analytical predictions from model flows, although, as discussed later, many of its magnetohydrodynamics (MHD) features have been discussed using kinematic or direct simulations of model situations. In the first versions of VKS experiment-VKS1, between 2000 and 2002 and VKS2, between 2005 and July 2006-all materials used had the same magnetic permeability $\left(\mu_{r} \simeq 1\right)$. However none of these configurations succeeded in providing dynamo action: the present paper analyzes dynamo regimes observed in the VKS2 setup when the liquid sodium is stirred with pure soft-iron impellers $\left(\mu_{r}\right.$ $\sim 100$ ). Although the VKS2 dynamo is therefore not fully homogeneous, it involves fluid motions that are much more fluctuating than in previous experiments. The role of the ferromagnetic impellers is at present not fully understood, but is thoroughly discussed in the text.

Below, we will first describe the experimental setup and flow features. We then describe and discuss in detail in Sec. II our observations of the dynamo generated when the impellers that drive the flow are in exact counter-rotation. In this case the self-sustained magnetic field is statistically stationary in time. We describe in Sec. III the dynamical re- 
gimes observed for asymmetric forcing conditions, i.e., when the impeller rotation rates differ. Concluding remarks are given in Sec. IV.

\section{B. The VKS experiment}

The VKS experiment studies MHD in a sodium flow generated inside a cylinder by counter-rotation of impellers located near the end plates. This flow has two main characteristics which have motivated its choice. First, its timeaveraged velocity field has both helicity and differential rotation, as measured in Refs. 21-23, two features that have long been believed to favor dynamo action. ${ }^{2}$ Second, the von Kármán geometry generates a very intense turbulence in a compact volume of space. ${ }^{24-31}$

The topology of the time-averaged velocity field has received much attention because it is similar to the $s_{2} t_{2}$ flow considered in a sphere by Dudley and James. ${ }^{32}$ This topology generates a stationary kinematic dynamo ${ }^{33-35}$ whose main component is a dipole perpendicular to the rotation axis (an " $m=1$ " mode in cylindrical coordinates). The threshold, in magnetic Reynolds number, for kinematic dynamo generation is very sensitive to details of the mean flow such as the poloidal to toroidal velocity ratio, ${ }^{33-35}$ the electrical boundary conditions, ${ }^{34-38}$ or the details of the velocity profile near the impellers. ${ }^{38}$ Specific choices in the actual flow configuration have been based on a careful optimization of the kinematic dynamo capacity of the VKS mean flow. ${ }^{22,35}$ The above numerical studies do not take into account the fully developed turbulence of the von Kármán flows, for which velocity fluctuations can be on the order of the mean. In actual laboratory conditions the magnetic Prandtl number of the fluid is very small (about $10^{-5}$ for sodium) so that the kinetic Reynolds number must be indeed huge in order to generate even moderate magnetic Reynolds numbers. A fully realistic numerical treatment of the von Kármán flows is out of reach. The influence of turbulence on the dynamo onset has thus been studied using model behaviors ${ }^{39-41}$ or numerical simulations in the related Taylor-Green (TG) flow. ${ }^{42-44}$ These fluctuations may have an ambivalent contribution. On the one hand, they may increase the dynamo threshold compared to estimates based on kinematic dynamo action but, on the other hand, they could also favor the dynamo process. In particular, TG simulations have shown that the threshold for dynamo action may be increased when turbulent fluctuations develop over a well-defined mean flow, ${ }^{45-47}$ but that it saturates to a finite value for large kinetic Reynolds numbers. Initial simulations of homogeneous isotropic random flows ${ }^{48,49}$ have suggested a possible runaway behavior, although a saturation of the threshold has been observed in the most recent simulations. ${ }^{50}$ Finally, we note that many dynamo models used in astrophysics actually rely on the turbulence-induced processes. We will argue here that the VKS dynamo generated when the impellers are counterrotated at equal rates is a dynamo of the $\alpha$ - $\omega$ type.

Similar $s_{2} t_{2}$ geometries are being explored in experiments operated by Lathrop and co-workers ${ }^{51-53}$ at University of Maryland College Park and by Forest and co-workers ${ }^{54-56}$ at University of Wisconsin, Madison. Sodium flows are gen-
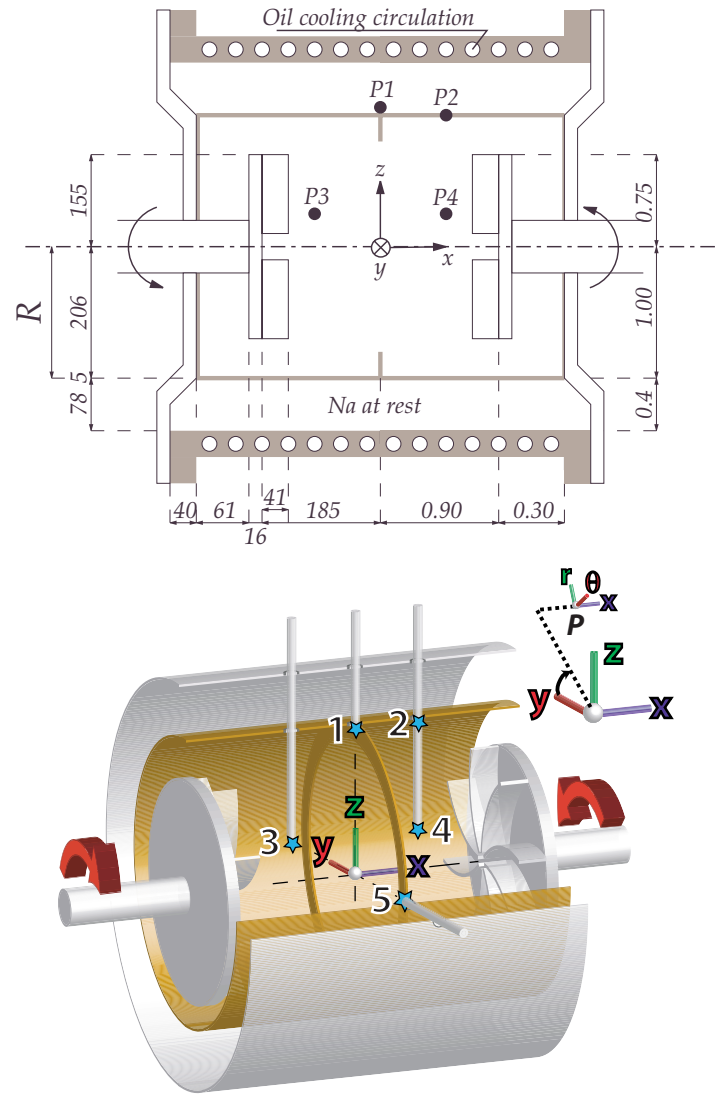

FIG. 1. (Color online) Experimental setup. Note the curved impellers, the inner copper cylinder which separates the flow volume from the blanket of surrounding sodium, and the thin annulus in the midplane. Also shown are the holes through which the 3D Hall probes are inserted into the copper vessel for magnetic measurements. Location in $(r / R, \theta, x / R)$ coordinates of available measurement points: $P_{1}(1, \pi / 2,0), \quad P_{2}(1, \pi / 2,0.53)$, $P_{3}(0.25, \pi / 2,-0.53), P_{4}(0.25, \pi / 2,0.53)$, and $P_{5}(1, \pi, 0)$ (see Table I for details concerning available measurements in different experimental runs). When referring to the coordinates of magnetic field vector $\mathbf{B}$ measured in the experiment at these different points, we will use either the Cartesian projection $\left(B_{x}, B_{y}, B_{x}\right)$ on the frame $(x, y, z)$ or the cylindrical projection $\left(B_{r}, B_{\theta}, B_{x}\right)$ on the frame $(r, \theta, x)\left(B_{\theta}=-B_{y}\right.$ and $B_{r}=B_{z}$ for measurements at points $\mathrm{P} 1, \mathrm{P} 2$, and $\mathrm{P} 3$ ). We will use in figures the following color code for the magnetic field components: axial $(x)$ in blue, azimuthal $(y)$ in red, and radial $(z)$ in green.

erated in spherical vessels with a diameter ranging from 30 $\mathrm{cm}$ to $1 \mathrm{~m}$. Changes in Ohmic decay times using externally pulsed magnetic fields in Maryland have again suggested that the dynamo onset may significantly depend on fine details of the flow generation. ${ }^{52,53}$ Analysis of induction measurements, i.e., fields generated when an external magnetic field is applied in Madison have shown that turbulent fluctuations can induce an axial dipole and have suggested that a bifurcation to a transverse dipole may proceed via an on-off scenario, ${ }^{55,57,58}$ as observed also in the Bullard-von Kármán experiment. $^{59}$

\section{Setup details}

In the VKS2 experiment, the flow cell is enclosed within a layer of sodium at rest (Fig. 1). The flow is generated by rotating two impellers with radius of $154.5 \mathrm{~mm}, 371 \mathrm{~mm}$ apart in a thin cylindrical copper vessel, $2 R=412 \mathrm{~mm}$ in inner diameter, and $524 \mathrm{~mm}$ in length. The impellers are 
fitted with eight curved blades with height of $h=41.2 \mathrm{~mm}$; in most experimental runs, the impellers are rotated so as the blades move in a "nonscooping" direction, defined as the positive direction by arrows in Fig. 1. The impellers are driven up to typically $27 \mathrm{~Hz}$ by motors with $300 \mathrm{~kW}$ available power.

When both impellers rotate at the same frequency $F_{1}$ $=F_{2}$, the complete system is symmetric with respect to any rotation $\mathcal{R}_{\pi}$ of $\pi$ around any radial axis in its equatorial plane $(x=0)$. Otherwise, when $F_{1} \neq F_{2}$, the system is not $\mathcal{R}_{\pi}$-symmetric anymore. For simplicity, we will also refer to symmetric/asymmetric system or forcing.

The kinetic and magnetic Reynolds numbers are

$$
\operatorname{Re}=K 2 \pi R^{2} F / \nu
$$

and

$$
R_{m}=K \mu_{0} \sigma 2 \pi R^{2} F,
$$

where $F=\left(F_{1}+F_{2}\right) / 2, \nu$ is the kinematic viscosity of sodium, $\mu_{0}$ is the magnetic permeability of vacuum, $\sigma$ is the electrical conductivity of sodium, and $K=0.6$ is a coefficient that measures the efficiency of the impellers for exact counterrotation $F_{1}=F_{2}=F .^{21-23,35}$ Re can be increased up to 5 $\times 10^{6}$, and the corresponding maximum magnetic Reynolds number is $R_{m}=49$ (at $120^{\circ} \mathrm{C}$ ). These definitions of Re and

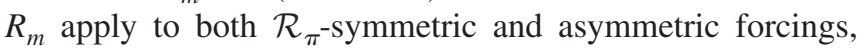
but the $K$-prefactor correctly incorporates the efficiency of the forcing only in the symmetric case. Reynolds numbers may also be constructed either on $F_{1}$ and $F_{2}$, e.g., $R_{m, i}$ $=K \mu_{0} \sigma 2 \pi R^{2} F_{i}$. All these definitions collapse for exact counter-rotating, i.e., $\mathcal{R}_{\pi^{-}}$-symmetric regimes.

The flow is surrounded by sodium at rest contained in another concentric cylindrical copper vessel, $578 \mathrm{~mm}$ in inner diameter and $604 \mathrm{~mm}$ long. An oil circulation in this thick copper vessel maintains a regulated temperature in the range of $110-160^{\circ} \mathrm{C}$. Note that there are small connections between the inner sodium volume and the outer sodium layer (in order to be able to empty the vessel) so that the outer layer of sodium may be moving; however, given its strong shielding from the driving impellers, we expect its motion to be weak compared to that in the inner volume. Altogether the net volume of sodium in the vessel is 1501 -more details are given in Fig. 1.

In the midplane between the impellers, one can attach a thin annulus-inner diameter of $350 \mathrm{~mm}$ and thickness of 5 $\mathrm{mm}$. Its use has been motivated by observations in water experiments, ${ }^{21-23,35}$ showing that its effect on the mean flow is to make the midplane shear layer steadier and sharper. All experiments described here are made with this annulus attached; in some cases we will mention the results of experiments made without it in order to emphasize its contribution.

Finally, one important feature of the VKS2 experiment described here is that the impellers that generate the flow have been machined from pure soft iron $\left(\mu_{r} \sim 100\right)$. One motivation is that self-generation has not been previously observed for identical geometries and stainless steel impellers. Several studies have indeed shown that changing the magnetic boundary conditions can change the threshold for dynamo onset. ${ }^{60,61}$ In addition, kinematic simulations have shown that the structure of the sodium flow behind the driving impellers may lead to an increase in the dynamo threshold ranging from $12 \%$ to $150 \% ;^{38}$ using iron impellers may protect induction effects in the bulk of the flow from effects occurring behind the impellers. As described in detail below, this last modification allows dynamo threshold to be crossed. We will further discuss the influence of the magnetization of the impellers on its onset.

Magnetic measurements are performed using threedimensional (3D) Hall probes and recorded with a National Instruments PXI digitizer. We use both a single-point (three components) Bell probe (hereafter labeled G probe) connected to its associated gaussmeter and a custom-made array where the 3D magnetic field is sampled at ten locations along a line (hereafter called SM array), every $28 \mathrm{~mm}$. The array is made from Sentron 2SA-1M Hall sensors and is air cooled to keep the sensors' temperature between 35 and $45^{\circ} \mathrm{C}$. For both probes, the dynamical range is $70 \mathrm{~dB}$, with an ac cutoff at $400 \mathrm{~Hz}$ for the gaussmeter and $1 \mathrm{kHz}$ for the custom-made array; signals have been sampled at rates between 1 and $5 \mathrm{kHz}$.

During runs, we have also recorded other more global data such as torque/power measurements: ${ }^{28,62}$

(a) The temperature of sodium in the vessel. This is important because the electrical conductivity $\sigma$ of sodium varies in the temperature range of our experiments. In addition, it gives an alternate way of varying the magnetic Reynolds number: one may operate at varying speed or varying temperature. We have used

$\sigma(T)=10^{8} /(6.225+0.0345 T) \mathrm{S} \mathrm{m}^{-1}$

for $T \in[100,200]{ }^{\circ} \mathrm{C}$.

(b) The torque delivered by the electrical supply of the driving motors. It is estimated from outputs of the UMV4301 Leroy-Sommer generators, which provide an image of the torque fed to the motors and of their velocity (regulated during the runs). We stress that this is an industrial indicator-not calibrated against independent/reference torque measurements.

\section{DYNAMO GENERATION, SYMMETRIC DRIVING $F_{1}=F_{2}$}

\section{A. Self-generation}

On September 19th, 2006, we observed the first evidence of dynamo generation in the VKS experiment. ${ }^{16}$ The flow was operated with the thin annulus inserted and the magnetic field was recorded in the midplane using the $G$-probe located flush with the inner cylinder (location P1 in Fig. 1).

As the rotation rate of the impeller $F=F_{1}=F_{2}$ is increased from 10 to $22 \mathrm{~Hz}$, the magnetic field recorded by the probe develops strong fluctuations and its main component (in the azimuthal direction at the probe location) grows and saturates to a mean value of about $40 \mathrm{G}$, as shown in Fig. 2(a). This value is about 100 times larger than the ambient magnetic field in the experimental hall, from which the flow volume is not shielded. The hundred-fold increase is also one order of magnitude larger that the induction effects and field 
(a)

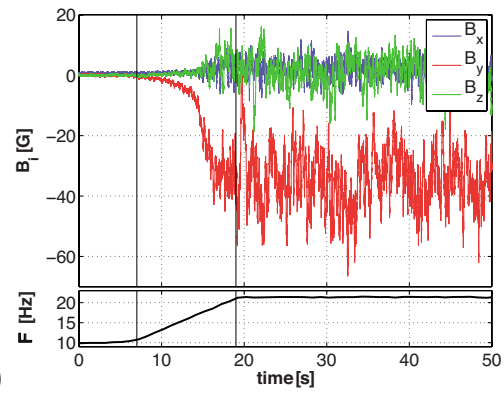

(b)
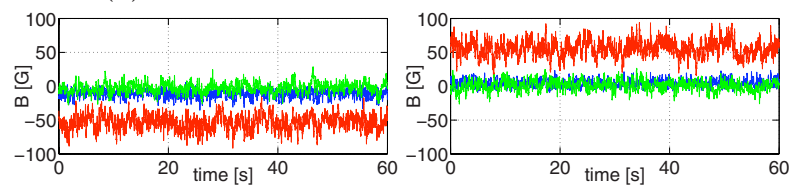

FIG. 2. (Color online) Three components of the magnetic field generated by dynamo action at $F_{1}=F_{2}$ measured at point P1 in the experiment VKS2g. (a) Growth of the magnetic field as the impellers' rotation rate $F$ is increased from 10 to $22 \mathrm{~Hz}$. (b) Two independent realizations at same frequency above threshold showing opposite field polarities.

amplification previously recorded in the VKS experiment with externally applied magnetic field, either homogeneously over the flow volume ${ }^{19}$ or localized at the flow boundary. ${ }^{63}$ We interpret this phenomenon as evidence of dynamo selfgeneration in VKS2.

The dynamo field is statistically stationary: the components of the magnetic field measured at the boundary or within the flow display large fluctuations around a nonzero mean value as seen in Fig. 2. When the experiment is repeated, one observes that the azimuthal field at the probe location can grow with either polarity [Fig. 2(b)] as is expected from the $\mathbf{B} \rightarrow-\mathbf{B}$ symmetry of the MHD equations.

The most salient features of this turbulent dynamo have been reported in a letter. ${ }^{16}$ They are given in more detail in this section. We first show that the amplitude of the magnetic field behaves as expected for a supercritical (but imperfect) bifurcation; we discuss the structure of the neutral mode and the influence of the magnetization of the iron impellers.

\section{B. Bifurcation}

Figure 3(a) shows the evolution of the magnetic field measured at P1 when the magnetic Reynolds number varies. When $R_{m}$ exceeds $R_{m}^{c} \sim 32$, the magnetic field grows spontaneously. In the experiment $R_{m}$ can be varied either by changing the rotation rate of the impellers, or the working temperature of the sodium in the vessel, and both effects need to be taken into account when building the bifurcation curves in Fig. 3. In other words, the growth of the magnetic field is governed by the proximity to threshold in magnetic Reynolds number $\left(R_{m}-R_{m}^{c}\right)$ and not by the single hydrodynamic (rotation rate $F$ ) or magnetic (diffusivity $1 / \mu_{0} \sigma$ ) parameters.

The magnetic field grows from very small values (the induction from the ambient field) and saturates to a value which increases as a function of $\left(R_{m}-R_{m}^{c}\right)$. The behavior is identical when the magnetic field grows in one direction or the other. In a canonical supercritical bifurcation, one would expect a scaling region $B_{\text {sat }} \propto\left(R_{m}-R_{m}^{c}\right)^{a}$ with $a=1 / 2$. Our results are compatible with such a behavior, although a better

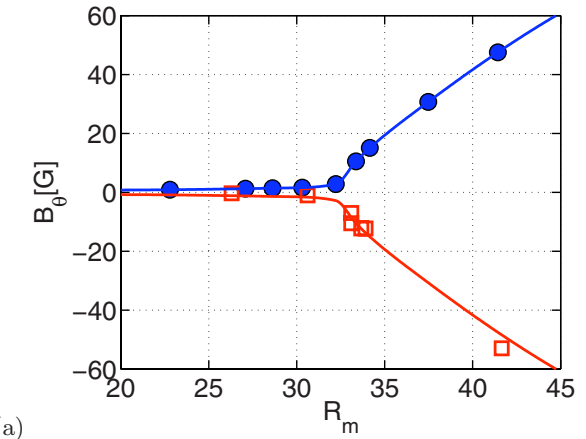

(a)

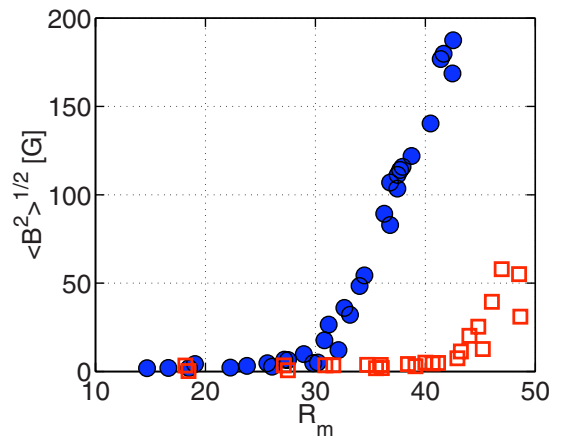

FIG. 3. (Color online) Bifurcation curves. (a) Azimuthal field measured at P1 for VKS2h, growing with either polarity (only measurements with initially demagnetized impellers are shown here). The solid lines correspond to a best fit with a scaling behavior $B_{\theta} \sim\left(R_{m}-32\right)^{0.77}$ above threshold. (b) Magnetic field amplitude $\left\langle B^{2}\right\rangle^{1 / 2}=\sqrt{B_{x}^{2}+B_{y}^{2}+B_{z}^{2}}$ at P3 for VKS2i. Impellers are counter-rotating at equal rotation rates in the positive direction shown in Fig. 1 (closed blue circles) or in the opposite direction, i.e., with the blades on the impellers moving in a scooping or negative direction (open red squares). Changes in the efficiency of the stirring are taken into account in the definition of $R_{m} ; R_{m}=K \mu_{0} \sigma R^{2} F$ with $K=K_{+}=0.6$ in the normal, positive direction of rotation and $K=K_{-}=0.7$ in the opposite direction.

fit to the data is $B_{\theta} \sim\left(R_{m}-32\right)^{0.77}$ for the azimuthal component of the magnetic field (the largest component at the measurement point). More measurements and better statistics will, of course, be needed to get a precise scaling behavior; we also stress that one should proceed with caution when ascribing scaling exponents to this bifurcation. The instability grows over a strongly noisy background (the flow hydrodynamic turbulence) and no rigorous theory has been developed in this context- the existence of anomalous exponents is an open question.

Another effect that influences the precise shape of the bifurcation is the magnetic behavior of the iron impellers. As seen in Fig. 2, the dynamo field has a strong azimuthal component. The iron impellers have a direction of easy magnetization in the same direction. The coercitivity of pure iron is weak and the remanent magnetization is of the order of $H_{c}$ $\sim 100 \mathrm{~A} / \mathrm{m}$, while the saturation flux density exceeds $20000 \mathrm{G}$. This magnetization of the impellers induces a bias in the dynamo instability. Experimentally we have observed that once the dynamo grows above the onset with a given polarity of the azimuthal field, then it will always start in the same direction when the flow is driven successively below and above onset. The reverse polarity, as shown in Figs. 2 and 3, are only observed when the impellers have been demagnetized (at least partially) by being exposed to an oscillatory field. The imperfection induced in the bifurcation is 


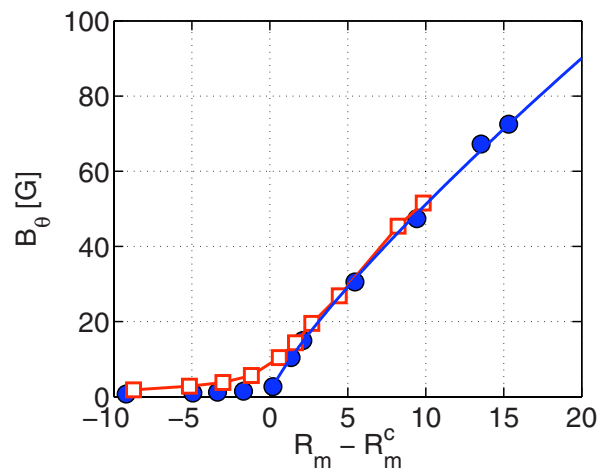

FIG. 4. (Color online) Bifurcation diagrams measured for the azimuthal component at P1 in VKS2h. The curve with closed blue circles is built when increasing $F$ with demagnetized impellers and crossing the dynamo threshold for the first time. Successive cycles in $R_{m}$ across the bifurcation threshold all lie on the curve with open red square symbols.

shown in Fig. 4. The (circles/blue) curve is obtained when starting the experiment from demagnetized impellers and the bifurcation is crossed for the first time. The (squares/red) curve is obtained for further successive variations in the magnetic Reynolds number below and above the onset. As shown in Ref. 64, the rounding off of the bifurcation curve can be explained in a minimal model for which the magnetization is coupled to a supercritical bifurcation. Other effects that could be a priori associated with the ferromagnetism of the driving impellers have not been observed. For instance, after the bifurcation has been crossed for the first time, we have not detected any hysteretic behavior of the dynamo onset, and the smooth continuous increase in the field with $\left(R_{m}-R_{m}^{c}\right)$ is at odds with the behavior of solid rotor dynamos. ${ }^{3,4}$ Disk dynamos with imposed velocity would also saturate to a value directly fixed by the maximum available mechanical power, in contrast to our observations (cf. Sec. II E).

One last observation strongly ties the self-generated magnetic field with the hydrodynamics of the flow. Features of the flow (mean geometry and fluctuations) can be changed when the driving impellers are counter-rotated in either direction (labeled + or - ), or the thin annulus may be inserted or not in the midplane (called the $\mathrm{w}$ and w/o configurations). ${ }^{22,23,31}$ Bifurcation curves for the $+\mathrm{w}$ and $-\mathrm{w}$ cases are shown in Fig. 3(b). When the blades of the impellers are rotated in "scooping" $(-)$ or nonscooping $(+)$ directions, we note that the dynamo threshold is changed from $F=16 \mathrm{~Hz}$ in the + w case to $F=18 \mathrm{~Hz}$ in the - w/o case, with corresponding power consumption changing from 40 to 150 $\mathrm{kW}$. Taking into account the change in the efficiency with which the fluid is set into motion, the threshold varies from $R_{m}^{c}=32$ to $R_{m}^{c} \sim 43$ in terms of the integral magnetic Reynolds number. For the $+\mathrm{w} / \mathrm{o}$ case (not shown), the onset is again $R_{m}^{c} \sim 30$. However, for the - w/o case we have reached $R_{m} \sim 49$ without observing any dynamo generation. At this stage a detailed comparison of the hydrodynamics and MHD of the four $(+,-, \mathrm{w}, \mathrm{w} / \mathrm{o})$ flows is not available, but a water prototype allows us to measure the ratio $\xi$ of the mean poloidal velocity to the mean toroidal velocity. ${ }^{21-23,34,35}$ For all flows, we get $\xi=0.8$ except for the $(-$ w/o $)$ flow where $\xi$

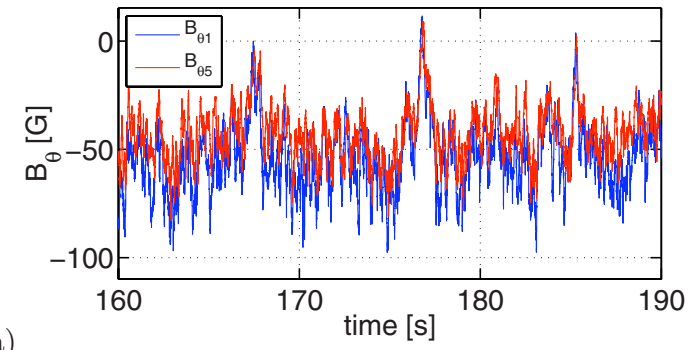

(a)

(b)

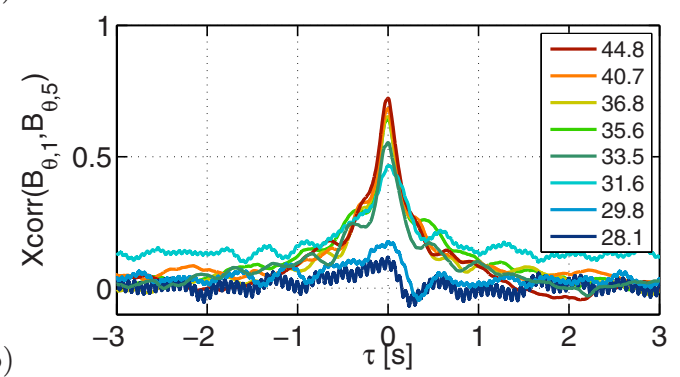

FIG. 5. (Color online) Magnetic field measured at points P1 and P5 in VKS2h: (a) Time traces of the azimuthal magnetic field components. (b) Cross correlation function of the two signals for increasing magnetic Reynolds numbers in the range of $28-45$.

$=0.5$. In fact, Ravelet and co-workers ${ }^{22,35}$ showed that for the VKS2 configuration, $\xi=0.8$ is optimal for kinematic dynamo, while $\xi=0.5$ does not support kinematic dynamo action at all. One may suppose that this ratio has an effect on the fully turbulent dynamo action. Furthermore, strong large scale fluctuations of the flow may hinder dynamo action. Such quantities can be evaluated using the ratio $\delta(t)$ of the instantaneous to the time-averaged kinetic energy densities. ${ }^{31}$ Within the four considered flows, the $(-$ w/o) flow presents the highest level for both the mean value and the variance of $\delta(t),{ }^{31}$ respectively, $10 \%$ and $33 \%$ more than the dynamogenerating flow that fluctuates most $(+\mathrm{w} / \mathrm{o})$.

\section{Geometry of the dynamo field}

A central issue is the structure of the magnetic field which is self-sustained above threshold. In order to address it, we have inserted probes and probe arrays at several locations within the sodium flow (cf. Fig. 1). Due to our limited sampling in space, we do not fully map the neutral mode; we only obtain indications of its topology. We describe them in detail in this section; measurements reported concern the $+\mathrm{w}$ flow case.

In VKS2h runs, simultaneous measurements at points $\mathrm{P} 1$ and P5 were performed so that the field is sampled in the midplane at two locations flush with the inner copper cylinder and at a $\pi / 2$ angle. As can be observed in the time signals shown in Fig. 5(a), the azimuthal components of the magnetic fields at these two points vary synchronously. The maximum of the autocorrelation function [Fig. 5(b)] occurs for a zero time lag; its value is less than 0.2 below the dynamo onset, jumps to 0.5 at the threshold, and saturates to values around 0.7 for $R_{m}$ values above 36 .

In Fig. 5(b), we have plotted times in seconds and observe that all the curves above the dynamo onset almost collapse. This behavior persists if time is made nondimensional 


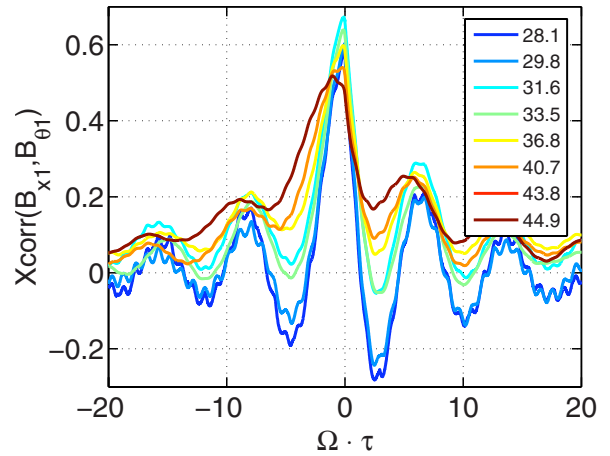

FIG. 6. (Color online) Cross correlation function of the axial component and azimuthal component of the field measured inside the flow for increasing magnetic Reynolds numbers in the range of 28-45. Measurements are done at point P1 in VKS2h.

using the diffusion time $\mu_{0} \sigma R^{2}$ but does not persist when using the impeller rotation rate $F$ (not shown). This shows that the dynamics is linked to a global magnetic mode rather than controlled by hydrodynamic processes. Another observation is that there is also a strong correlation between the axial and azimuthal components of the magnetic field at a given location. Figure 6 shows that its maximum exceeds 0.5 at all $R_{m}$, and that it occurs for a time delay increasing with $R_{m}$.

In VKS2i runs, the Hall-probe array was set within the bulk of the flow, nearer to one impeller-point P3. For several values above onset $\left(R_{m}=33.9,35.8,41.4\right)$, we observe in Fig. 7 that the normalized field profiles along the array nicely superimpose. Near the rotation axis, the main component is axial; further away the azimuthal component dominates. Outside of the flow volume (beyond the inner copper cylinder) the axial component direction is opposite to that in the center. In the same experiment, the single-point probe was set nearer to the other impeller (symmetrically with respect to the midplane, and close to the rotation axis, point P4). The field measured there is mainly axial with the same direction as at P3. Below threshold, the induction profiles (due to the

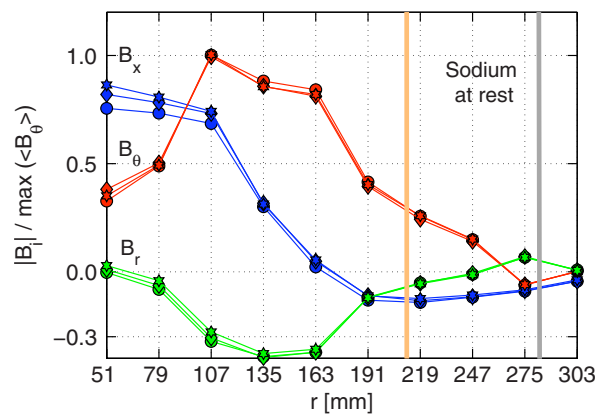

FIG. 7. (Color online) Radial profiles of magnetic field. VKS2i measurements with the probe array inserted at P3. Measurements for $+w$ flows at $R_{m}=33.9,35.8,41.4$, above the dynamo onset. The magnetic field components are normalized by the largest value of the $\theta$-component (azimuthal direction): axial $(x)$ is blue, azimuthal $(\theta)$ is red, and radial $(r)$ is green. The thick vertical line around the seventh sensor $(r \sim 211 \mathrm{~mm})$ indicates the position of the inner copper shell; the tenth sensor at $r=303 \mathrm{~mm}$ is located into the outer copper wall of the vessel.
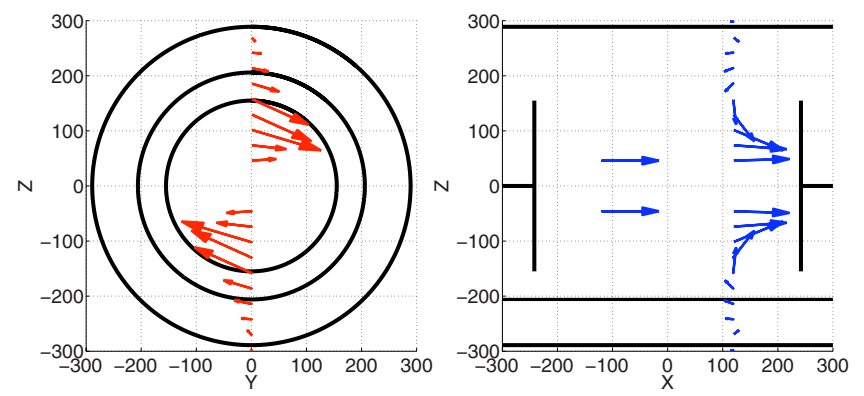

(a)

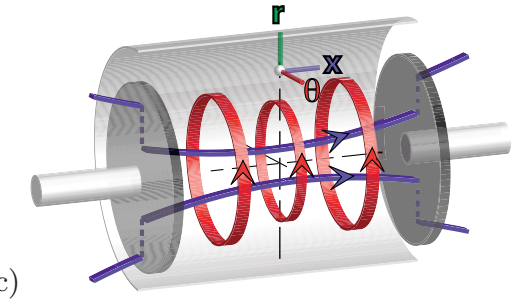

(b)

FIG. 8. (Color online) Geometry of the dynamo mode. The magnetic field amplitudes measured along the probe array in Fig. 7 is represented by arrows: (a) toroidal component, (b) poloidal component, and (c) proposed dipole structure for the neutral mode.

presence of the Earth's field) have the same geometry: the favored dynamo mode is the most efficient with respect to induction.

The combined results of the two sets of experiments are shown in Figs. 8(a) and 8(b) - the toroidal and poloidal components are displayed and axisymmetry is assumed. The simplest geometry of the magnetic field corresponds to an axisymmetric field whose axial component has one direction at the center of the cylinder and a reversed direction outside, together with a strong azimuthal component-sketched in Fig. 8(c). The dynamo field generated in the VKS2 experiment thus involves a strong axial dipolar component. The determination of its precise geometry (associated higher modes and their relative amplitudes) needs more precise measurements. However, it strongly differs from the prediction of kinematic calculations based on the topology of the mean von Kármán flow ${ }^{35,36}$ which tends to favor a transverse dipole. Invoking Cowling's theorem, ${ }^{2}$ the axisymmetric nature of the dynamo fields implies that it has not been generated by the mean flow motions alone. However, an axial dipole is expected for an $\alpha-\omega$ dynamo. The differential rotation in von Kármán flows can generate an azimuthal field from a large scale axial field by torsion of the field lines-the $\omega$ effect. ${ }^{65}$ If an $\alpha$ effect takes place, it can produce an azimuthal current from the azimuthal magnetic field component and, in turn, this current will generate an axial magnetic field. One then has a dynamo loop-back cycle that can sustain an axial dipole. For the $\alpha$ component, several mechanisms have been proposed. For an axisymmetric mean flow, it has been shown that nonaxisymmetric fluctuations can generate such an alpha effect provided that they have no mirror symmetry. ${ }^{66}$ A scenario was proposed in Ref. 64 using the helicity of the flow ejected by the blades of the driving impellers - this flow is nonaxisymmetric with azimuthal wavenumber $m=8$, helical, and of much larger amplitude and coherence than small scale turbulent fluctuations. In this 
case, an azimuthal loop of magnetic field crosses an array of like-sign helical motions that can induce an azimuthal current. ${ }^{20,67-69}$ Numerical integration of the mean field induction equation with an $\alpha$ effect localized in the vicinity of the impellers ${ }^{70}$ recently displayed the generation of an axial magnetic field. The authors of this study thus claim to show agreement with the scenario proposed in Ref. 64. In mean field theory, ${ }^{2,71}$ the most common source of $\alpha$ effect come from the small scale helicity of turbulence. However, the distribution of helicity in 3D turbulence remains a challenging question and measurements in model helical flows ${ }^{72,73}$ have failed to show evidence for this kind of contribution. As shown by induction measurements in Refs. 72 and 73, another possible contribution to the $\alpha$ effect lies in the spatial inhomogeneity of the turbulence intensity. As shown in Ref. 74 , there is a contribution to the mean-field alpha tensor coming from the inhomogeneity of turbulent fluctuations, with resulting electromotive force $\epsilon \sim(\mathrm{g} \cdot \boldsymbol{\Omega}) \mathbf{B}$, where $\boldsymbol{\Omega}$ is the flow vorticity and $\mathbf{g}$ is the normalized gradient of turbulent fluctuations, $\mathbf{g}=\left(\nabla u^{2}\right) / u^{2}$. It shows again that an azimuthal $B_{\theta}$ field generates a $j_{\theta}$ current and, therefore, an axial magnetic field. Distinguishing between these mechanismswhich may be linked - will need further measurements, ideally from nearby velocity and magnetic probes.

\section{Saturation}

The amplitude of the magnetic field at saturation is related to the way in which the growing Lorentz forces change the hydrodynamics of the flow. While the value of the threshold can be determined by studying the induction equation for a given velocity field-kinematic dynamo simulations - the saturation is governed by nonlinear effects and one must consider the coupled equations of MHD,

$$
\frac{\partial \mathbf{u}}{\partial t}+(\mathbf{u} \cdot \nabla) \mathbf{u}=-\nabla\left(\frac{p}{\rho}+\frac{B^{2}}{2 \mu_{0}}\right)+\nu \Delta \mathbf{u}+\frac{1}{\rho \mu_{0}}(\mathbf{B} \cdot \nabla) \mathbf{B},
$$

$$
\frac{\partial \mathbf{B}}{\partial t}+(\mathbf{u} \cdot \nabla) \mathbf{B}=(\mathbf{B} \cdot \nabla) \mathbf{u}+\frac{1}{\mu_{0} \sigma} \Delta \mathbf{B}
$$

for divergence-free fields. Dimensionally, the value of the magnetic field at saturation can be written as

$$
B_{\text {sat }}=\rho \mu_{0} V^{2} f\left(R_{m}, P_{m}\right),
$$

where $f$ is a function of the magnetic Reynolds number $R_{m}$ and magnetic Prandtl number $P_{m}$ and $V$ is some characteristic velocity scale. Functional forms have been determined in limiting cases of laminar and turbulent dynamos. ${ }^{64,75,76}$ In the low (kinetic) Reynolds number limit, the Lorentz force balances the viscous term in the Navier-Stokes equation and one obtains ${ }^{75,76}$

$$
B_{\text {sat }}^{2} \propto \frac{\rho \nu}{\sigma R^{2}}\left(R_{m}-R_{m}^{c}\right) .
$$

In the high magnetic Reynolds number limit, the Lorentz force must be balanced by the nonlinear terms and one gets

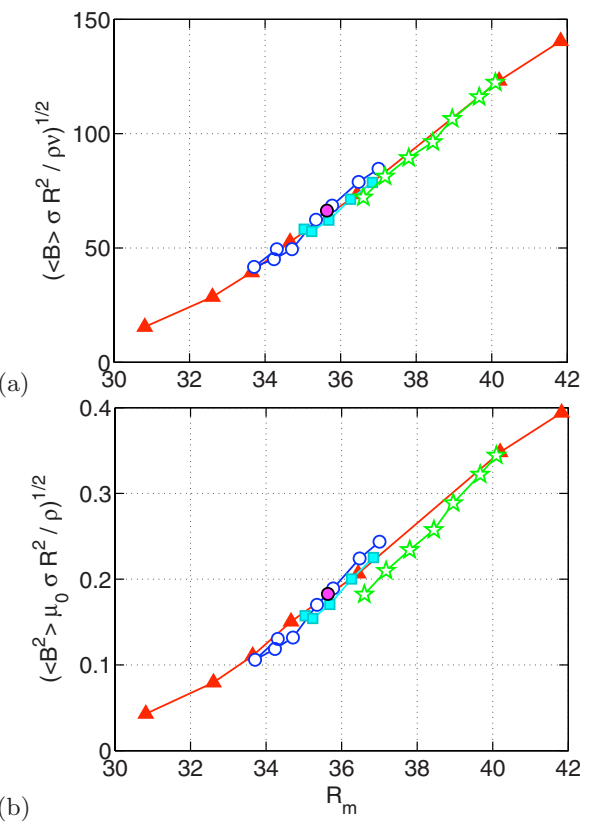

FIG. 9. (Color online) Laminar and turbulent scalings for the magnetic field intensity at saturation. Measurements are shown here for several rotation rates of the impellers and for different operating temperatures $T$; the magnetic Reynolds number $R_{m}$ is rescaled accordingly. Measurement at P1 in VKS2h, $\left\langle B^{2}\right\rangle^{1 / 2} \mid=\sqrt{B_{x}^{2}+B_{y}^{2}+B_{z}^{2}}$ : (a) laminar scaling [Eq. (5)] and (b) turbulent scaling [Eq. (6)]. Closed red triangles: $F=24 \mathrm{~Hz}, T=127 \rightarrow 143{ }^{\circ} \mathrm{C}$; closed cyan squares: $F=19 \mathrm{~Hz}, T=117 \rightarrow 137{ }^{\circ} \mathrm{C}$; open blue circles: $F$ $=19 \mathrm{~Hz}, \quad T=117 \rightarrow 145^{\circ} \mathrm{C}$; and open green stars: $F=21 \mathrm{~Hz}, \quad T=124$ $\rightarrow 152{ }^{\circ} \mathrm{C}$.

$$
B_{\text {sat }}^{2} \propto \frac{\rho}{\mu_{0} \sigma^{2} R^{2}}\left(R_{m}-R_{m}^{c}\right) .
$$

Both possibilities are shown in Fig. 9, in which the magnetic Reynolds number has been varied either by changing the rotation rate of the driving impellers or by changing the operating temperature and, hence, the sodium's electrical conductivity. Judging from the linearity of the graphs, both seem plausible; however, one may note that the prefactor in the laminar scaling is very large. In other words, a laminar approach, quite doubtful given the high turbulence level in the sodium flow, also underestimates the saturated field by almost two orders of magnitude. We favor the turbulent scaling and note at this point that the fluctuations play an essential role for the generation of the dynamo and that inertial terms in the Navier-Stokes equation control the nonlinear saturation.

\section{E. Power consumption issues}

We now turn to the power needed to drive the flow in its steady state, below, or above threshold. Let us call $\mathbf{u}_{0}(\mathbf{r}, t)$ the velocity field during the linear growth of the dynamo instability, and $P_{0}$ the mechanical power needed to drive this velocity field in a stationary forcing. Given the very high kinetic Reynolds number of the experimental flow, one expects the turbulent scaling $P_{0} \propto \rho L^{2} U_{0}^{3}$, where $L$ and $U_{0}$ are characteristic length and velocity scales. We define the di- 


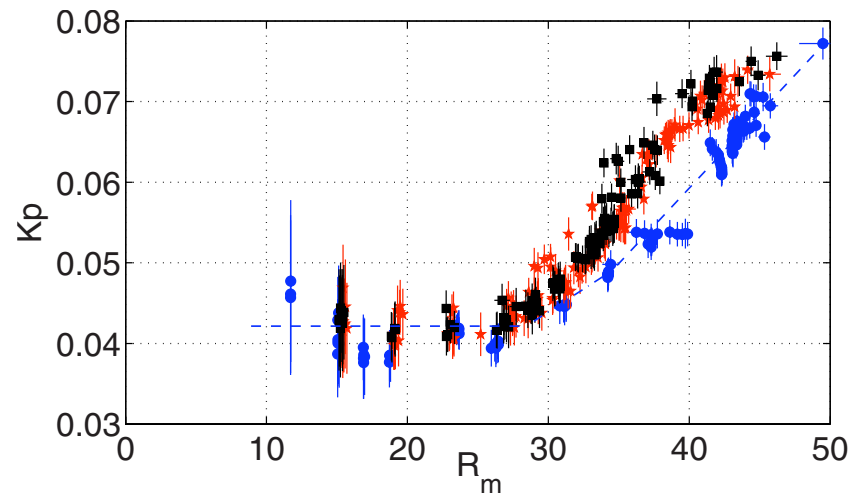

FIG. 10. (Color online) Evolution with $R_{m}$ of the power number defined in Eq. (7) and measured from the drives of the motors. The blue circles (and dashed line as an eye guide) are from nondynamo runs (VKS2f) with stainless steel impellers. The red stars (VKS2g) and black squares (VKS2h) come from two dynamo runs, with same geometry but with soft-iron impellers and also without any magnetic probes in the flow bulk.

mensionless constant (or power number) $K_{p}$, using the torques $\left(\Gamma_{1}, \Gamma_{2}\right)$ or the total power $\left(P_{0}\right)$ delivered by the driving motors, as

$$
K_{p}=\frac{1}{2} \frac{\Gamma_{1}+\Gamma_{2}}{\rho R^{5}(2 \pi F)^{2}}=\frac{1}{2} \frac{P_{0}}{\rho R^{5}(2 \pi F)^{3}},
$$

where we have used $R$ as the characteristic length scale and $U_{0}=2 \pi R F$ as the associated velocity scale. The $1 / 2$ factor in the definition corresponds to the consumption per motor, as used in reports of water-prototype studies. ${ }^{21,22,35}$ Please note that the two formulas in Eq. (7) are only equal because $F_{1}$ $=F_{2}$. When a dynamo is generated, the velocity field in the saturated state is $\mathbf{u}_{B}(\mathbf{r}, t)$. The mechanical power is now $P_{B}$ and it must feed both the hydrodynamic flow and the magnetic field: $P_{B}=P_{B}^{\mathrm{hydro}}+P_{B}^{\mathrm{mag}}$. The field $\mathbf{u}_{B}$ differs from $\mathbf{u}_{0}$ because the nonlinear saturation has modified the flow. A likely possibility is that the turbulent fluctuations have been changed, ${ }^{6475-77}$ and this would also change the mean flow via the Reynolds stress tensor. The power needed in order to sustain the magnetic field must be estimated from

$$
P_{B}^{\mathrm{mag}}=\int \frac{j^{2}}{\sigma} d^{3} x=\int \frac{(\nabla \times \mathbf{B})^{2}}{\mu_{0}^{2} \sigma} d^{3} x,
$$

where $j$ is the current density. With the limited measurements at this stage of the experiment, we cannot resolve independently $P_{B}^{\text {hydro }}$ and $P_{B}^{\mathrm{mag}}$, and we can only use the industrial output of the motors drives as an indicator of $P_{0}$ and $P_{B}$.

The dimensionless power number is shown in Fig. 10 for three sets of experiments. The blue circles correspond to measurements made when driving the flow with stainless steel impellers, a case for which dynamo action was not observed. A first observation is that the expected turbulent scaling $-K_{p}$ independent of Re or $R_{m}$-is observed only for $R_{m}<30(F<16 \mathrm{~Hz})$. The $K_{p}=0.045$ value is in agreement with measurements made in scaled-down water prototypes. ${ }^{21-23,31}$ Above $R_{m}=30$, some modification must occur because the flow power consumption climbs, in nondimensional units, from $K_{p}=0.045$ to $K_{p} \sim 0.065$, when $R_{m}$ $>40(F>24 \mathrm{~Hz})$. This phenomenon, not understood at

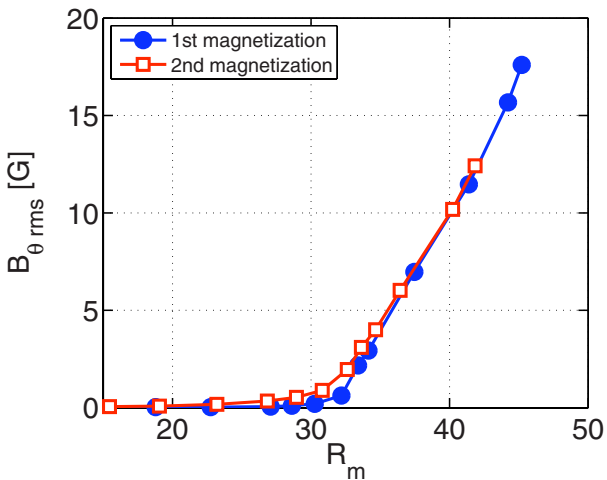

(a)

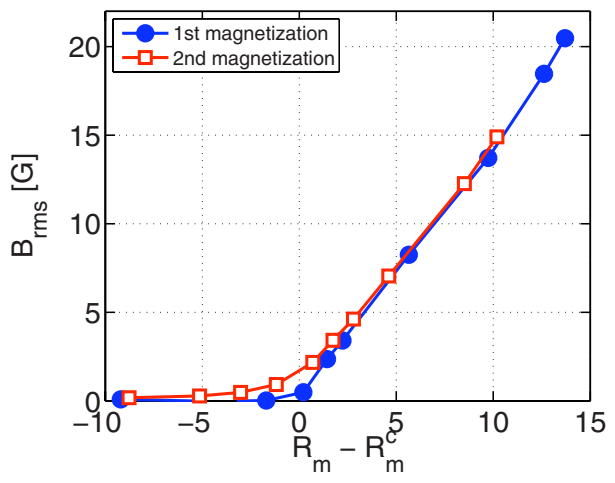

FIG. 11. (Color online) Evolution with $R_{m}$ of rms values of the magnetic field at saturation. Measurements at point P1 in VKS2h. (a) Azimuthal component $B_{\theta \mathrm{rms}}$. (b) Magnetic field amplitude $B_{\text {rms. }}$. The closed blue circles corresponds to a first run with almost demagnetized impellers (see text) and the open red squares to the subsequent runs.

present, has to be related to the hydrodynamics of the flow because it is observed in nondynamo experiments. However, for identical rotation rates of the impellers, we measure on average that $K_{p}$ (dynamo) is typically $10 \%$ larger than $K_{p}$ (nondynamo).

\section{F. Fluctuations}

All results presented so far have concerned the average — in time — of the magnetic field recorded by the probes. This section discusses features of the rather large fluctuations about these mean values-as can be seen in Fig. 2. One first observation is that the rms amplitudes in each of the components of the magnetic field increase sharply at dy-

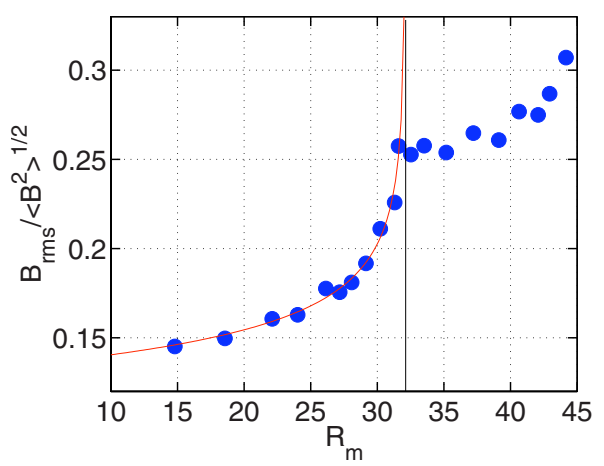

FIG. 12. (Color online) Evolution of the ratio of rms fluctuations to second moment of the magnetic field at point P1. 


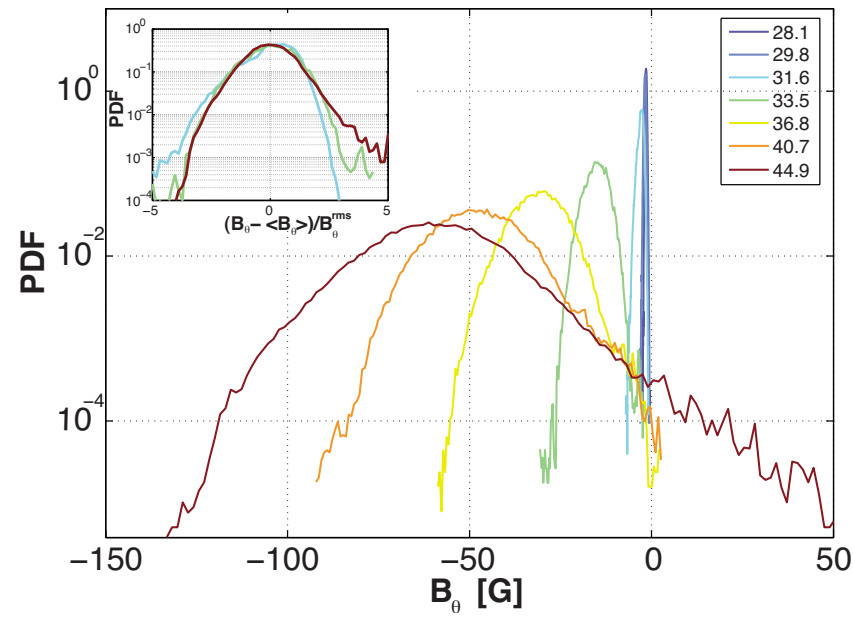

FIG. 13. (Color online) PDFs of local fluctuations of $B_{\theta}$. Measurement at point P1, VKS2h. Magnetic Reynolds numbers are given in the legend and profiles below onset cannot be distinguished. Inset: PDFs for centered and normalized variables.

namo onset, as shown in Fig. 11. This increase defines the same threshold $R_{m}^{c} \sim 32$, as estimated from the behavior of the mean values.

Another interesting observation concerns the evolution of the ratio of rms to second moment. It increases very sharply near onset and then much less rapidly above (cf. Fig. 12). The transition actually allows a determination of the threshold, again at $R_{m}^{c} \sim 32$. From a practical point of view, the sharp nonlinear increase in the fluctuations may be a good indicator that one is nearing dynamo onset.

We now study the fluctuations in time of the dynamo field recorded at a fixed location. Each component has instantaneous fluctuations that deviate significantly from the mean values. Figure 13 shows the probability density functions (PDFs) for the azimuthal component measured by the gaussmeter at P1. While sharply peaked about $B_{\theta}=0$ below threshold, the distributions become wide above threshold. At the highest $R_{m}$ values, one notes the development of a weak exponential tail to low values with occurrence of events with the reversed polarity. These events, which may also be spatially localized within the flow, are not able to reverse the dynamo. When normalized and centered (inset in Fig. 13), the distributions are still essentially Gaussian in the vicinity of the threshold and there is no evidence that the bifurcation proceeds via on "on-off" scenario. ${ }^{55,57-59}$ Gaussian distributions were also observed for most induction measurements carried out in the VKS1 setup, ${ }^{19}$ so that self-excitation has not induced significant changes in behavior here-in agreement with measurements in the Karlsruhe dynamo. ${ }^{10}$

The fluctuations whose statistics are given above occur over a wide range of time scales. The time spectra give the distribution of energy among these scales. Since the fluctuations of the magnetic field measured at a given location in the experiment are expected to be sensitive to the local turbulence intensity, we discuss in the following the magnetic energy spectra measured at different locations and at different magnetic Reynolds numbers.

We report in Fig. 14 the magnetic spectra measured above the dynamo threshold at different distances from the axis of the experiment along a radius passing through point P3. We observe a clear dependence of the global shape of the spectra depending on the location. The spectra exhibit ranges to which one may want to ascribe traditional turbulence scaling behavior:

(a) For $f \leqq F / 10$, the spectra are essentially flat; note however a broad frequency peak around $f_{\text {low }} \sim F / 10$ (we will see further that $\left.f_{\text {low }} \sim 0.14 F\right)$.

(b) In the range $F / 10 \leqq f \leqq 4 F$, the spectra are consistent with power laws: $f^{-1}$ for the azimuthal and the radial components and $f^{-5 / 3}$ for the axial component.

(c) At larger frequencies, the magnetic energy decays rapidly within a short range $(4 F \leqq f \leqq 8 F)$ possibly consistent with a $f^{-11 / 3}$ decay, also known to exist in numerous induction experiments with liquid metals ${ }^{19,65}$ and an even steeper decay (close to $f^{-6}$ ) at larger frequencies.

As we study signals from sensors at increasing radial distances from the axis, these regimes disappear. Harmonics locked to the impellers' rotation rate become visible: harmonic 8 is particularly intense for sensors at $r \lesssim 215 \mathrm{~mm}$, inside the inner copper vessel. This is very likely to be related to the fact that the impellers are fitted with eight blades and that those sensors intercept the centrifugal flow ejected at the periphery by the blades. As we move into the layer with sodium at rest $(r \gtrsim 210 \mathrm{~mm})$, the global energy of the
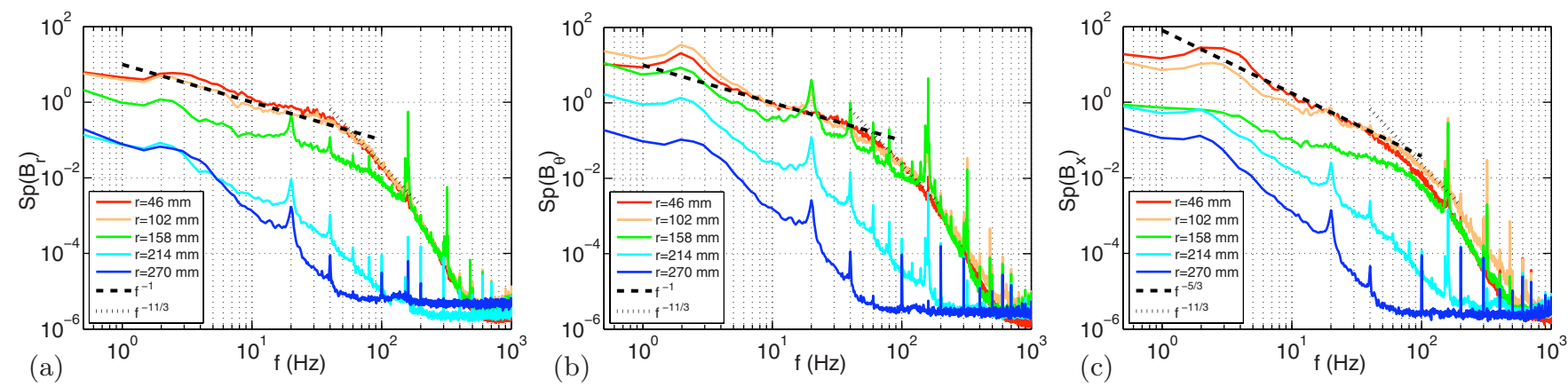

FIG. 14. (Color online) Time spectra of magnetic field fluctuations measured with the probe array at point P3 (location of the innermost sensor) in VKS2i. Different curves on each plot correspond to different sensor depth, labeled in the legend by their distance $r$ to the rotation axis. The rotation frequency of the impellers is $20 \mathrm{~Hz}$ : (a) Axial component, (b) azimuthal component, and (c) radial component. 


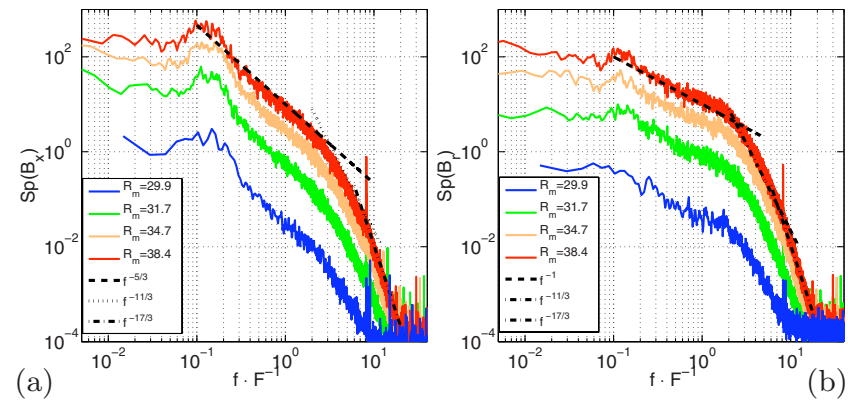

FIG. 15. (Color online) Time spectra of magnetic field fluctuations measured for different values of $R_{m}$ at point P4 during VKS2i: (a) axial component and (b) radial component.

fluctuations significantly decreases; a decay due to the presence of ohmic dissipation remains visible as well as the first harmonics of the impellers' rotation rate.

We now discuss the evolution of spectra with $R_{m}$, measured at the innermost location within the flow (Fig. 15). The different power law regimes remain, with their exponents unchanged for all values of the magnetic Reynolds number. We find that the transition between the different regimes is locked to forcing frequency $F$. The low frequency peak is systematically present and centered around $f_{\text {low }} \sim 0.14 F$ regardless of the Reynolds number. The $f^{-1}$ regime, which remains present for the azimuthal and radial component, may be interpreted as $1 / f$ noise due to the presence of several characteristic time scales in the flow. At larger frequencies, above the magnetic diffusion cutoff, the $f^{-11 / 3}$ regime is expected, under a Taylor hypothesis, from the balance in the induction equation between the magnetic induction termwith a fully turbulent velocity field - and the Ohmic dissipation term. The shortness of the $f^{-11 / 3}$ regime that we observe and the steeper decrease observed above the eighth harmonic can be attributed to a spatial filtering due to the dimension $l_{0} \sim 3 \mathrm{~cm}$ of the tube containing the probe. The spatial integration of magnetic fluctuations at the scale $l_{0}$ attenuates the spatial magnetic spectra by a factor $k^{-2}$, which under a Taylor hypothesis, should lead in the frequency spectral domain to a $f^{-17 / 3} \sim f^{-5.7}$ regime consistent with the steep energy decrease measured for large frequencies fluctuations. In this scenario the cutoff frequency between the $f^{-11 / 3}$ and the $f^{-17 / 3}$ regimes is proportional to $l_{0} v$, with $v$ the characteristic local velocity at the probe location. This is consistent with the observation that the transition is locked to the impellers' rotation rate.

Finally, we discuss measurements taken in the midplane, flush with the lateral wall, which have different characteristics. Figure 16 shows the evolution of spectra as the magnetic Reynolds number is increased. The three components have a similar behavior [Fig. 16(a)]. One notes a net magnetic energy increase as the dynamo threshold is crossed. At all magnetic Reynolds number, the spectra exhibit a low frequency peak $f_{\text {low }} \sim 0.14 F$ and a peak at a frequency corresponding to the rotation rate of the impellers, also present in the measurements at points P3 and P4 previously discussed. Although the evidence of power law dependence of the spectrum is again restricted to relatively short ranges, different regimes may be identified:

(a) At low frequencies (typically $f \lesssim f_{\text {low }}$ ) a slow decay with an exponent $\alpha_{1}$ changing from $\alpha_{1} \sim 0$ below threshold to $\alpha_{1} \sim-0.5$ above threshold.

(b) At intermediate frequencies (typically $f_{\text {low }} \lesssim f \leqq F$ ) the energy decay is faster with an exponent $\alpha_{2}$, changing from $\alpha_{2} \sim-2$ below threshold to $\alpha_{2} \sim-2.5$ above threshold.

(c) In the high frequency range (typically $f \gtrsim F$ ), as shown in the semilog representation in Fig. 16(c), the spectra are better described by an exponential decay $\exp \left(-f / f_{\exp }\right)$. We find that $f_{\exp }$ is of the order of $1.4 F$.

\section{DYNAMICAL REGIMES WITH ASYMMETRIC FORCING $F_{1} \neq F_{2}$}

\section{A. Asymmetric flow forcing}

In this section, we report results obtained when the impellers rotate at different frequencies $\left(F_{1} \neq F_{2}\right)$. Different sets of parameters can be used to describe these forcing configurations: (i) $F_{1}$ and $F_{2}$, the two impellers frequencies; (ii) $F$ $=\left(F_{1}+F_{2}\right) / 2$ the mean frequency and

$$
\Theta=\left(F_{1}-F_{2}\right) /\left(F_{1}+F_{2}\right)
$$

the nondimensional difference; and (iii) any other combinations of $F_{1}$ and $F_{2}$. In a half-scale water model experiment, it has been shown from torque measurements ${ }^{21}$ that there are strong similarities between the von Kármán flow forced by
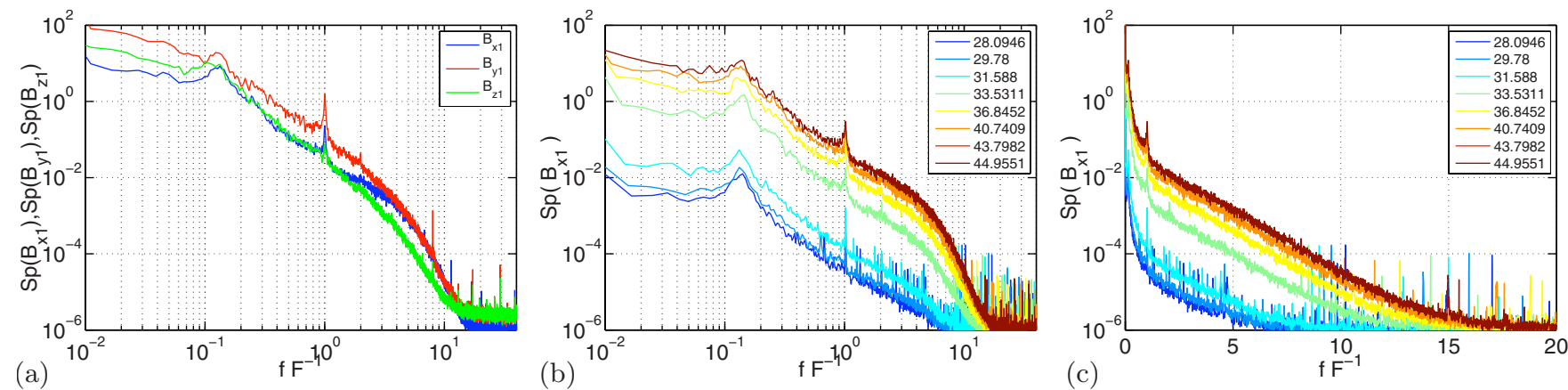

FIG. 16. (Color online) Time spectra of magnetic field fluctuations at point P1. (a) Three components, $R_{m} \sim 40$; [(b) and (c) $] \log$-log plot and semilog plots of $B_{x}$ spectra for increasing $R_{m}$ values. 


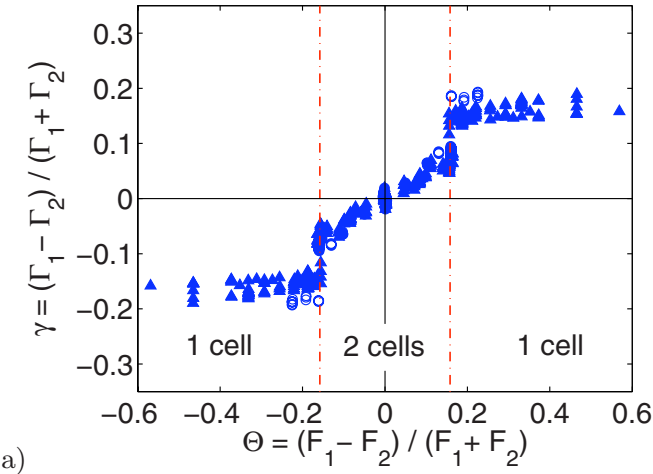

(a)

(b)

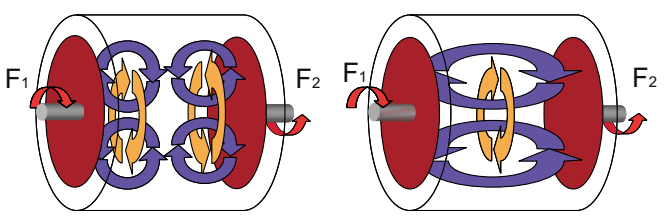

(c)

FIG. 17. (Color online) (a) Measurement of the reduced dimensionless torque $\gamma=\left(\Gamma_{1}-\Gamma_{2}\right) /\left(\Gamma_{1}+\Gamma_{2}\right)$ vs $\Theta=\left(F_{1}-F_{2}\right) /\left(F_{1}+F_{2}\right)$. Data are from VKS2g (open circles) and VKS2h (closed triangles), i.e., without probes in the flow bulk which strongly affect $\gamma$ in the one-cell regimes. Data have been symmetrized. (b) Schematic configuration of the mean flow in the two-cell regime for small $\Theta\left(F_{1} \simeq F_{2}\right)$, as measured in water. (c) Schematic configuration of the mean flow in the one-cell regime for large $\Theta\left(F_{1} \gg F_{2}\right)$.

impellers rotating respectively at $F_{1}$ and $F_{2}$ in the laboratory frame or by impellers rotating at $\left(F_{1}+F_{2}\right) / 2$ in a frame rotating at $\left(F_{1}-F_{2}\right) / 2$. More recently, stereoscopic particle image velocimetry measurements have confirmed this finding. ${ }^{23}$ It is thus convenient to choose $F$ and $\Theta$ as control parameters since $F$ appears as the effective forcing shear in the rotating frame and $|\Theta|$ measures the relative importance of the global rotation to shear.

These asymmetric regimes have been studied in water experiments allowing a good understanding of the hydrodynamic bifurcations of the flow when breaking the forcing symmetry. ${ }^{21,22,78}$ When increasing or decreasing one of the impeller rotation rates from a symmetric configuration, the cell closer to the fastest impeller grows in size and the shear layer is translated toward the slowest impeller. When only one impeller rotates, the flow is made of one single pumping cell rotating with the stirring impeller-similar to the $s_{1} t_{1}$ flow. ${ }^{32}$ The evolution between these extreme cases (generally referred to as the "turbulent bifurcation") depends on the impeller and vessel geometry: In some cases, the transition between the two extreme flow topologies (two symmetric cells versus one single cell) is smooth and continuous, but it might also be sudden, ${ }^{31,78}$ leading to first order transitions in any order parameter chosen to describe it (average position of the shear layer, torque delivered by the motors, etc.).

Figure 17 shows the evolution of the reduced dimensionless torques

$$
\gamma=\left(\Gamma_{1}-\Gamma_{2}\right) /\left(\Gamma_{1}+\Gamma_{2}\right)
$$

delivered by the motors as a function of $\Theta$. One can see the sudden transition for $|\Theta|=\Theta_{c} \simeq 0.16$, corresponding, e.g., to $F_{1}=22 \mathrm{~Hz}$ and $F_{2} \simeq 15.8 \mathrm{~Hz}$. At this point the flow transits between one-cell and two-cell topologies. ${ }^{22,31,78}$ The transitions are very sharp and the corresponding hysteresis domains are very narrow.

\section{B. A variety of regimes}

We first report observations of dynamo regimes when varying the frequency of rotation of the impellers. Not all possible combinations have been explored-Fig. 18 summarizes the visited regions of parameter space. They are plotted in terms of the experimental control parameters $\left(F_{1}, F_{2}\right)$ and also as a function of the differential rotation $\Theta$ and of an average magnetic Reynolds number based on the global rotation rate $F=\left(F_{1}+F_{2}\right) / 2$. In Fig. 18(b), symmetry between the positive and negative values of $\Theta$ has been assumed and observed dynamical regimes will be discussed according to this hypothesis, with $F$ and $|\Theta|$ being the control parameters.

For $R_{m}(F) \sim 35$, and for increasing $|\Theta|$, one gets successively-Fig. 18(b) — the following main regimes:

(1) Stat1: $|\Theta|<0.09$; stationary dynamos of the same type as observed for the symmetric $\Theta=0$ forcing. An example of time trace of the three components of magnetic field measured at P1 is shown in Fig. 19(a).

(2) Limit cycle (LC): $|\Theta| \sim 0.1$; for several time-periodic regimes between the Stat1 and Stat2 states [Figs. 19(b) and 19(c)]. These regimes have been studied in detail in Ref. 18.
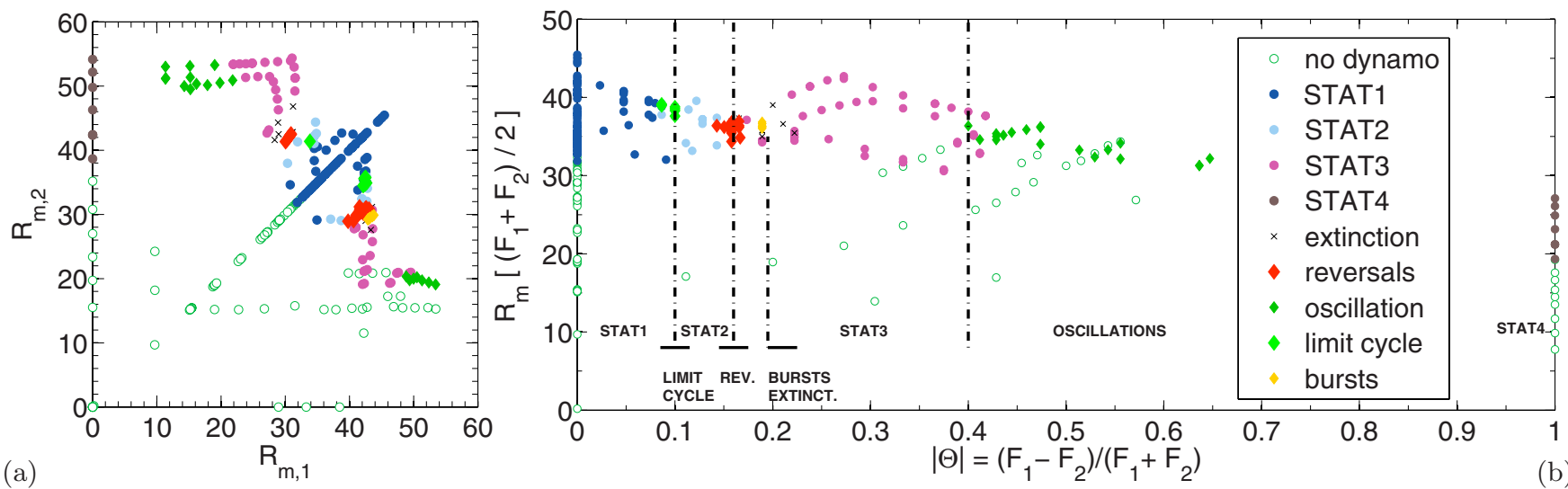

FIG. 18. (Color online) Parameter space and dynamo regimes for VKS2g, VKS2h, and VKS2i. (a) In the $\left(R_{m, 1}, R_{m, 2}\right)$ plane. (b) In the $\left[R_{m}(F),|\Theta|\right]$ plane, i.e., symmetrized data. 

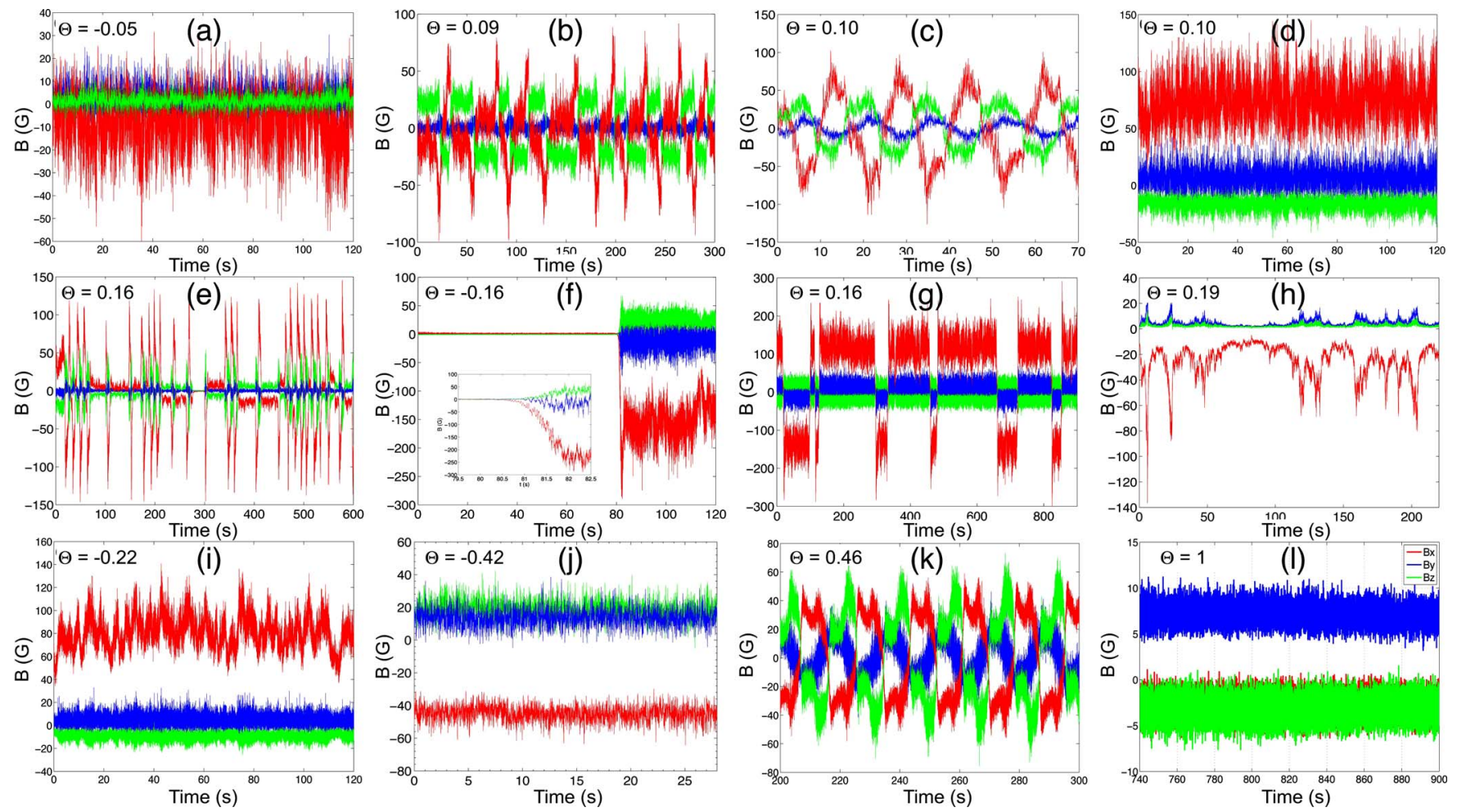

FIG. 19. (Color online) Examples of observed dynamo regimes for increasing values of $|\Theta|$. See text for details. Color code [see caption of Fig. 1 and legend of Fig. 2(a)] for the magnetic field components: axial $(x)$ in blue, azimuthal $(y)$ in red, and radial $(z)$ in green.

(3) Stat2: $0.11<|\Theta|<0.13$; stationary regimes [Fig. 19(d)].

(4) Reversal: $|\Theta| \in[0.15-0.18]$; reversing dynamos ${ }^{17,18}$ [Fig. 19(g)].

(5) Bursts-ext: $|\Theta| \sim 0.2$; regimes with a "bursting" dynamo field where the magnetic field has on average a low value with sudden "jerks" to much larger intensities [Figs. 19(e) and 19(h)]. In this region we have also observed cases where dynamo action was not observed within 3 minutes test periods, as well as cases when an initial dynamo decays to zero. They have been called "extinctions" [Figs. 19(f) and 25(d)].

(6) Stat3: $0.2<|\Theta|<0.4$; stationary dynamos [shown in Figs. 19(i) and 19(j)].

(7) Osc: $|\Theta|>0.4$; oscillatory dynamos. When increasing further the rotation component of the forcing, oscillatory dynamos are starting from the Stat3 stationary ones. An example is given in Figs. 19(k) and 27(a).

(8) Stat4: at $|\Theta|=1$, when the flow is driven by the rotation of only one impeller, one observes an abrupt bifurcation to a stationary dynamo of very low (finite) amplitude [Fig. 19(1)]. Note that intermediate forcing conditions $(0.6 \lesssim|\Theta|<1)$ are not reachable at present for mechanical reasons.

We note that at $R_{m}(F) \simeq 35$ a dynamo is always generated for all explored values of $\Theta$. $\Theta$ thus plays an important role in the selection of the dynamo regime; it does not choose it uniquely as we have observed in several cases that the dynamo at given values of $\left(F_{1}, F_{2}\right)$ depends on the path that has been followed.

\section{Stationary dynamo regimes, $\Theta \neq 0$}

Although a variety of dynamical regimes have been observed when the flow is driven with impellers counterrotating at different rates, a large fraction of the phase space is actually populated by stationary regimes (see Fig. 18). In future studies these regimes will need to be fully characterized in terms of mode geometry, type of dynamo bifurcation, etc. For the moment, we classify them in broad classes separated by time-dependent dynamos and consider the three cases for $|\Theta| \lesssim 0.6$. The first one [Stat1; Fig. 19(a) shows a typical time series, see also Fig. 2] appears at low values of $\Theta(|\Theta|<0.09)$. The second regime [Stat2; Fig. 19(d)] appears as one increases $\Theta(0.11<|\Theta|<0.13)$, but it is separated from the first by a small range of $\Theta$ with a reversing regime. The third stationary regime [Stat3; Fig. 19(j)] appears at larger values of $\Theta(0.2<|\Theta|<0.4)$. The main difference can be observed in the fluctuations, which are dominated by very slow oscillations, with periods of several seconds. This observation is confirmed in Fig. 20(a), showing the power spectrum density for the three stationary regimes. Note that the three spectra collapse when the frequency is rescaled by the mean rotation frequency of the impellers, $F=\left(F_{1}+F_{2}\right) / 2$, at least for the frequencies larger than $F$. The small scale fluctuations in the spectrum are thus set by the effective $R_{m}$, i.e., turbulence. In Fig. 20(b), the centered and normalized PDFs of the three regimes are presented and display a fairly Gaussian behavior, like in the case of exact counter-rotation (see Fig. 13). 

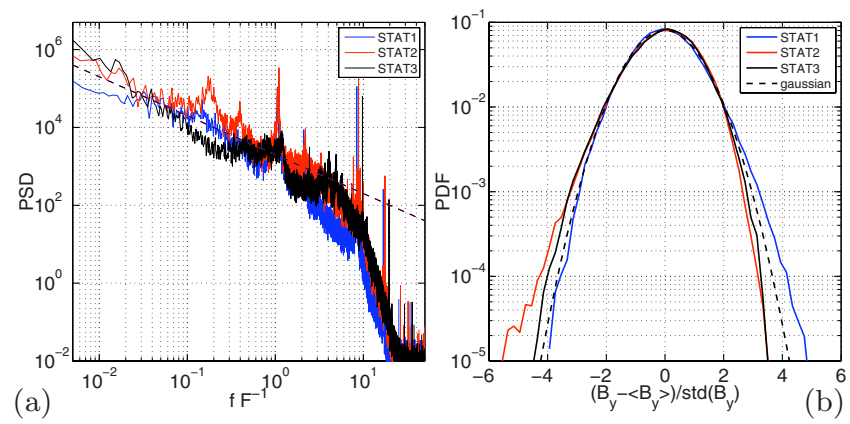

FIG. 20. (Color online) (a) Power spectrum density. Frequency is normalized by the average frequency of the impellers $F=\left(F_{1}+F_{2}\right) / 2$. The dashed line shows the -1 slope as a guide for the eye. (b) Centered PDF, normalized by its rms value. Quantities are computed from the azimuthal component in the time signal in Fig. 19 for the regimes Stat1, Stat2, and Stat3.

Although we have not systematically mapped the $\left(R_{m}, \Theta\right)$ plane, some observations can be made about the dynamo bifurcation in these regimes. For the Stat1 regime, the bifurcation seems supercritical with a threshold around $R_{m}=31$, similar to the counter-rotating case, but the dependence of the threshold on $\Theta$ has not been precisely determined. However as one proceeds to more asymmetric regimes, in the case of Stat2 and Stat3, the dependence of the dynamo field on the value of $\Theta$, as well as $R_{m}$, is more pronounced. A hysteretic behavior takes place, where the value of the magnetic field at saturation depends on the path followed.

In the limit of the flow being driven by the rotation of a single impeller (the other being kept at rest, i.e., $\Theta= \pm 1$ ), we observe an abrupt bifurcation to stationary dynamo Stat 4 . As the rotation rate of the impeller is increased above threshold, the amplitude of the magnetic field increases slightly to reach a maximum at $R_{m}=23 \mathrm{~Hz}$, then decreases to almost zero at the maximum rotation rate $\left(R_{m}=26\right)$. Such $R_{m}$ decrease in the dynamo is also observed when the equatorial annulus is removed but then the stationary regime exchanges stability with an oscillatory regime, with a bistability region. ${ }^{79}$

\section{Reversals}

Decreasing the frequency of one impeller from the stationary dynamo regime at $F_{1}=F_{2}=22 \mathrm{~Hz}$, we performed the first observation of reversals of the magnetic field in a fluid dynamo. ${ }^{17}$ These reversals at irregular time intervals are displayed in Fig. 21 for a sensor located at point P2. For each polarity, either positive or negative, the amplitude of the magnetic field has strong fluctuations, with a rms fluctuation level of the order of $20 \%$ of the mean. This level of fluctuation is due to the very intense turbulence of the flow, as the kinetic Reynolds number exceeds $10^{6}$. Reversals occur randomly and have been followed for up to $45 \mathrm{~min}$, i.e., 54000 characteristic time scales of the flow forcing.

The polarities do not have the same probability of observation. Phases with a positive polarity for the largest magnetic field component have, on average, longer duration $\left(\left\langle T_{+}\right\rangle=120 \mathrm{~s}\right)$ than phases with the opposite polarity $\left(\left\langle T_{-}\right\rangle\right.$ $=50 \mathrm{~s})$. This asymmetry can be due to the ambient magnetic field. Note however that the amplitude of the magnetic field

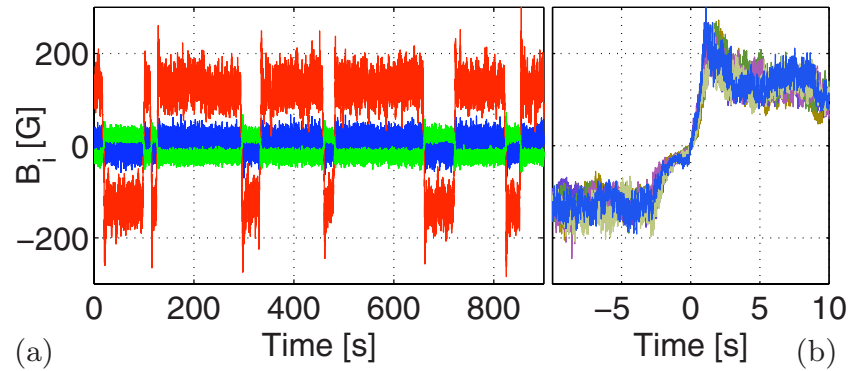

FIG. 21. (Color online) Reversals of the magnetic field generated by driving the flow with counter-rotating impellers at frequencies $F_{1}=16 \mathrm{~Hz}$ and $F_{2}$ $=22 \mathrm{~Hz}(\Theta=0.16, \mathrm{VKS} 2 \mathrm{~g})$. (a) Time recording of the three magnetic field components at P2: axial $(x)$ in blue, azimuthal $(y)$ in red, and radial $(z)$ in green [see legend of Fig. 2(a)]. (b) superimposition of the azimuthal component for successive reversals from negative to positive polarity together with successive reversals from positive to negative polarity with the transformation $B \rightarrow-B$. For each of them the origin of time has been shifted such that it corresponds to $B=0$.

is the same for both polarities. Standard deviations are of the same order of magnitude as the mean values, although better statistics may be needed to make these estimates more precise. The mean duration of each reversal, $\tau \sim 5 \mathrm{~s}$, is longer than MHD time scales: the flow integral time scale is of the order of the inverse of the rotation frequencies, i.e., $0.05 \mathrm{~s}$, and the Ohmic diffusive time scale is roughly $\tau_{\eta} \sim 0.4 \mathrm{~s}$.

When the amplitude of the magnetic field starts to decay, either a reversal occurs, or the magnetic field grows again with its direction unchanged. Similar sequences, called excursions or aborted reversals, ${ }^{80,81}$ are observed in recordings of the Earth's magnetic field. We have also observed that the trajectories connecting the symmetric states $\mathbf{B}$ and $-\mathbf{B}$ are quite robust despite the strong turbulent fluctuations of the flow. This is displayed in Fig. 21(a): the time evolution of reversals from positive to negative states can be neatly superimposed by shifting the origin of time such that $B(t=0)$ $=0$ for each reversal. Despite the asymmetry due to the Earth's magnetic field, negative-positive reversals can be superimposed in a similar way on positive-negative ones if $-B$ is plotted instead of $B$. For each reversal the amplitude of the field first decays exponentially. A decay rate of roughly $0.8 \mathrm{~s}^{-1}$ is obtained with a log-linear plot (not shown). After changing polarity, the field amplitude increases linearly and then displays an overshoot before reaching its statistically stationary state. The signal thus clearly breaks the time reversal symmetry, a common feature for many relaxation oscillations or heteroclinic cycles in low dimensional dynamical systems. A similar asymmetry in time, with a slow decay of the field amplitude followed by a fast recovery after changing sign, has been reported in recordings of the Earth magnetic field. $^{82}$

Magnetic field reversals have been found in the same region of parameter space during all the runs (VKS2g, VKS2h, and VKS2i). The characteristics described above have been found reproducible except the nearly perfect superimposition displayed in Fig. 21 (VKS2g). In the following runs where measurements were performed at P1 and P3, although a most probable trajectory clearly appears, some reversals follow a significantly different one between $\pm \mathbf{B}$. 


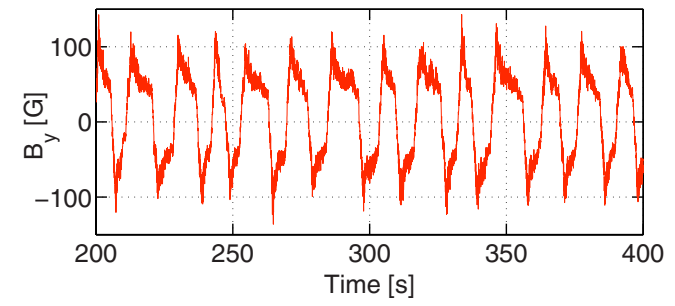

FIG. 22. (Color online) Nearly periodic reversals of the azimuthal magnetic field generated at $F_{1}=16 \mathrm{~Hz}$ and $F_{2}=22 \mathrm{~Hz}(\Theta=-0.16), \mathrm{VKS} 2 \mathrm{~h}$. Temperature of the outer copper cylinder $T=145{ }^{\circ} \mathrm{C}$ compared to about $120{ }^{\circ} \mathrm{C}$ for the regime with irregular reversals shown in Fig. 21.

Another difference concerns the synchronicity with which the three components of the magnetic field switch polarity; in the last runs (VKS2h and VKS2i), the different components have been found to vanish at slightly different times such that the norm of the magnetic field does not vanish during a reversal. These differences can be ascribed to small differences in the three runs. One possibility is that some features of the recordings of the magnetic field during reversals slightly depend on the location of the measurement point in the flow or to slight asymmetries of the setup revealed when $F_{1}<F_{2}(\mathrm{VKS} 2 \mathrm{~g})$ or $F_{1}>F_{2}(\mathrm{VKS} 2 \mathrm{~h})$. Furthermore, it is possible that measurements performed in the bulk in the last run (VKS2i) add disturbances to the flow that were not present in the first runs (VKS2g and VKS2h) for which the probes were flush to the lateral boundary. Another difference is related to the correlation of reversals with the global energy budget of the flow. The total power $P(t)$ delivered by the motors driving the flow has been shown to drop significantly below its average during reversals and excursions observed in the first set of runs (VKS2g) ${ }^{17}$ However, changes in polarity without noticeable modification of power have also been observed in most other runs.

We note that the randomness of the reversals strongly depends on the magnetic Reynolds number. For instance, starting from a regime with random reversals, we can reach a regime with nearly periodic noisy oscillations between $\pm \mathbf{B}$ by slightly changing the sodium temperature (see Fig. 22; this regime has been observed for $200 \mathrm{~s}$ corresponding to 14 periods $T \simeq 14 \mathrm{~s}$ ). Reversals can also bifurcate to different types of stationary dynamos or to chaotic regimes involving bursts (see below) by slightly varying the impeller frequencies.

\section{E. Bursts}

Bursts occur around $|\Theta| \sim 0.2$ when one impeller rotates at a rate roughly 1.5 times faster than the other one. For instance, when $F_{1}=22 \mathrm{~Hz}$ and $F_{2}=15 \mathrm{~Hz}$ we have observed the behavior shown in Fig. 23(a). The magnetic field develops "bursts" from a base state. In this base state [cf. Fig. 23(b)] the magnetic field takes values of the order of $20 \mathrm{G}$. This is much lower than most other stationary regimes, but nonetheless a dynamo generated field as its amplitude is over 40 times the ambient Earth's field. During the bursts, the dynamo field reaches high field values above $100 \mathrm{G}$, a level

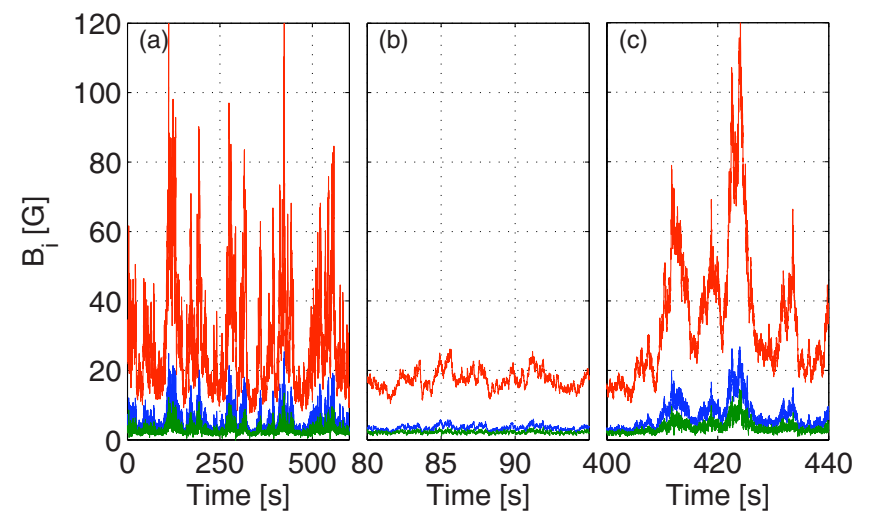

FIG. 23. (Color online) (a) Bursting regimes, VKS2h; $F_{1}=22 \mathrm{~Hz}, F_{2}$ $=15 \mathrm{~Hz}, \Theta=0.19$; three components of the magnetic field measured at point P1 [blue: axial component; red: minus azimuthal component; and green: radial component; see legend of Fig. 2(a)]. (b) and (c) are zoomed on, respectively, quiet periods and active bursts.

observed in other dynamical regimes such as reversals. Typical bursts, as in Fig. 23(c), have duration of about 2-10 s.

The correlation functions of the magnetic field also display this characteristic time. Figures 24(a) and 24(b) show the normalized correlation functions $\left\langle B_{i}(t) B_{j}(t+\tau)\right\rangle_{t}$ for the field components. A characteristic decay time of the order of $2.5 \mathrm{~s}$ is observed on all components. Such a characteristic time is long compared to the frequencies of the flow forcing $\left(F_{1}, F_{2}, F_{1} \pm F_{2}, \ldots\right)$ and even compared to the magnetic diffusion time over the radius of the vessel (of the order of 0.4 s). An exponential autocorrelation function is in agreement with a Lorentzian power spectrum as show in Fig. 24(c), where an $f^{-2}$ scaling can be observed for over 2 decades in the case of the azimuthal component of the magnetic field. The statistics of the fluctuations [Fig. 24(d)] displays an ex-

(a)
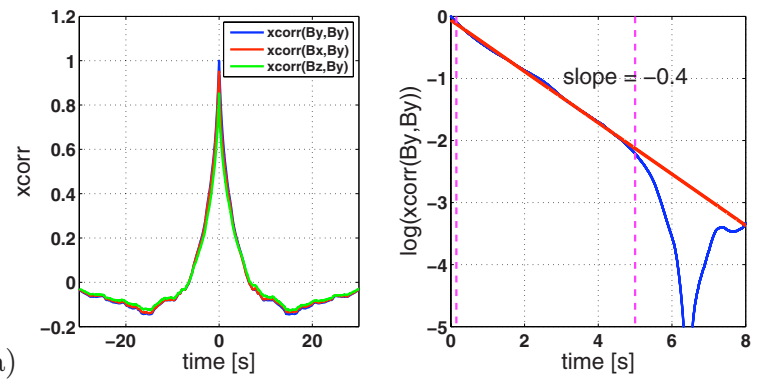

(c)
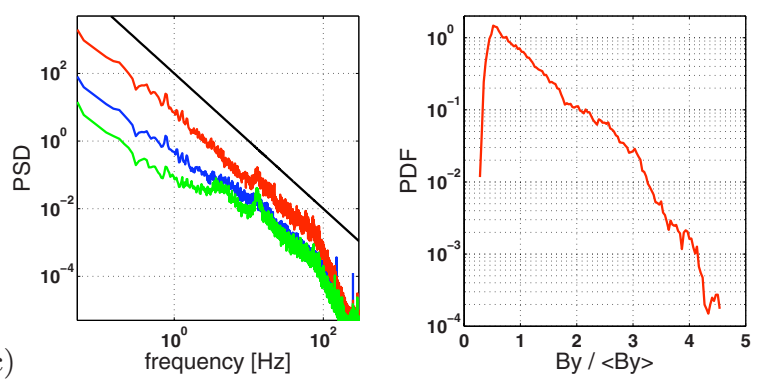

FIG. 24. (Color online) Bursting regimes, VKS2h; $F_{1}=22 \mathrm{~Hz}, F_{2}=15 \mathrm{~Hz}$, $\Theta=0.19$ [cf. Fig. 23]. (a) Time correlations of the magnetic field components. (b) log-linear plot of the autocorrelation of the largest field component $\left\langle B_{y}(t) B_{y}(t+\tau)\right\rangle_{t}$. (c) Corresponding time-power spectra-the black solid lines shows an $f^{-2}$ scaling. (d) PDF for $B_{y}(t)$. 

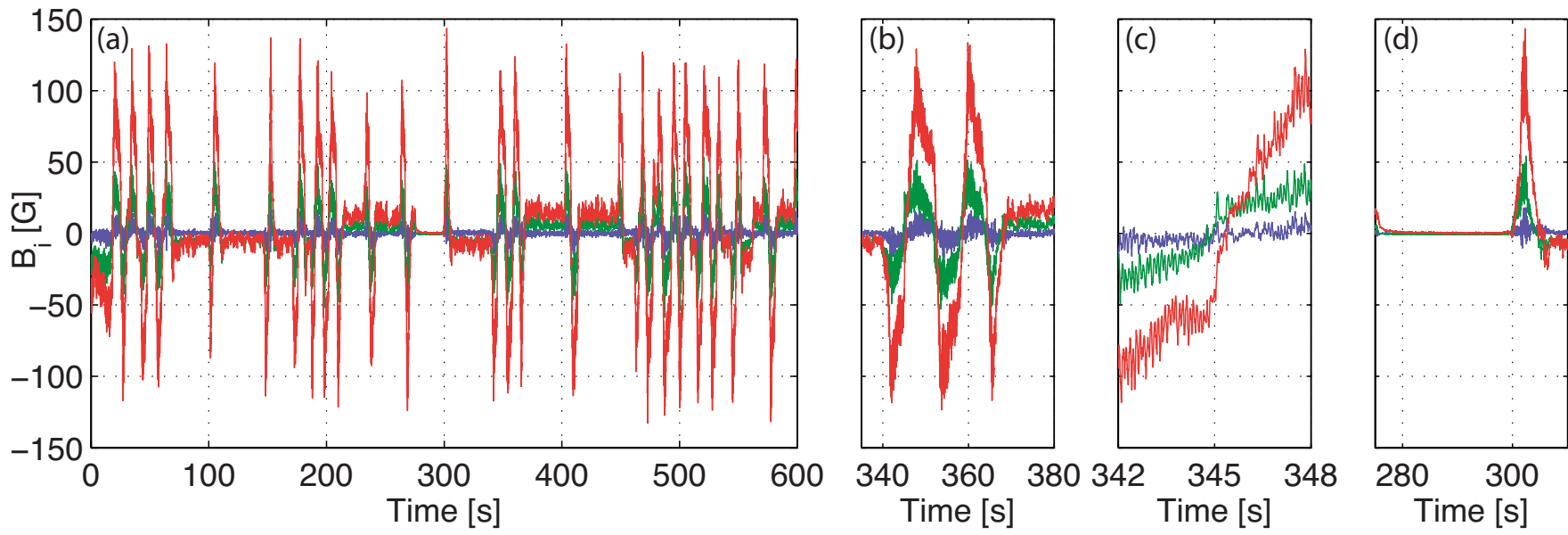

FIG. 25. (Color online) Bursting regimes, VKS2h; $F_{1}=21 \mathrm{~Hz}, F_{2}=15 \mathrm{~Hz}$, and $\Theta=0.167$. (a) Three components of the magnetic field measured at point P1 [blue: axial component; red: azimuthal component; and green: radial component; see legend of Fig. 2(a)]. (b) and (c) are further zoomed on the oscillations (note the change in polarity of the low state mode before and after the oscillations). (d) Zoom including the ending extinction around $t=300 \mathrm{~s}$.

ponential tail in the PDF, for the bursting events (large amplitudes). The distribution of interarrival times of bursts (not shown) is consistent with a homogeneous Poisson process, indicating that these bursting events are not intercorrelated.

When the velocity of the fastest impeller is slightly lowered, i.e., between the above described bursts domain and the reversals domain, another case of "bursting" events is observed. The dynamo transits from low amplitude states with either positive or negative polarity to the occurrence of oscillations, as seen in Fig. 25. The low amplitude states are again of the order 10-20 G, while the maximum amplitude reached when the oscillations are triggered is over $100 \mathrm{G}$, as in the oscillatory regimes discussed in Sec. III G. Note the extinction at $t \sim 300 \mathrm{~s}$, before the dynamo resumes its dynamics in the same regime.

Analysis of the correlation functions [Figs. 26(a) and 26(b)] shows a characteristic time of the order of $15 \mathrm{~s}$ with a dynamics such that the radial component of the local magnetic field is slightly ahead of the azimuthal and axial components. Two other peaks can be detected in the power spectra [Fig. 26(c)]: a shallow one at about $4 \mathrm{~Hz}$ and another, sharper, at $9 \mathrm{~Hz}-\mathrm{a}$ frequency clearly visible on the time signal in Fig. 25(c). The PDFs in Fig. 26(d) reveal the low field state and the larger amplitude during the oscillations. Note that PDFs are symmetric so that there is no preferred polarity in this regime.

\section{F. Extinction regimes}

In the range of $0.16 \leqq|\Theta| \lesssim 0.19$, i.e., where reversals, bursting reversals, and bursts occur, we also observe in few cases magnetic field extinctions (Ext). These extinctions consists of a total disappearance of all components of the dynamo field within the absolute precision due to the zero calibration of the probes, i.e., typically 0.5-2 G. They can clearly be distinguished from the low magnetic level $(\sim 20 \mathrm{G})$ regimes supporting the bursts [Fig. 23(b)]. Extinctions have been observed as transients or, if not transient, for 3 min test periods for the longest case. The most striking example is a spontaneous extinction observed during the bursting-reversal signal presented in Fig. 25 [see zoom in Fig. 25(d)]. Otherwise, extinctions have been observed when crossing the critical value $\Theta_{c} \sim 0.16$ where the flow topology changes (see Sec. III A) and may trace back for strong coupling when both flow and magnetic field reorganize. Figure 19(f) shows the reappearance of the dynamo at finite time from a transient extinction regime at $\Theta=-0.158$ : The initial condition is Stat3 regime at $\Theta=-0.22$ and the final one is the reversal regime at $\Theta=-0.158$. The dynamo disappears once the control parameter is changed and spontaneously reappears roughly $100 \mathrm{~s}$ later through exponential growth [inset in Fig. 19(f)].

(a)
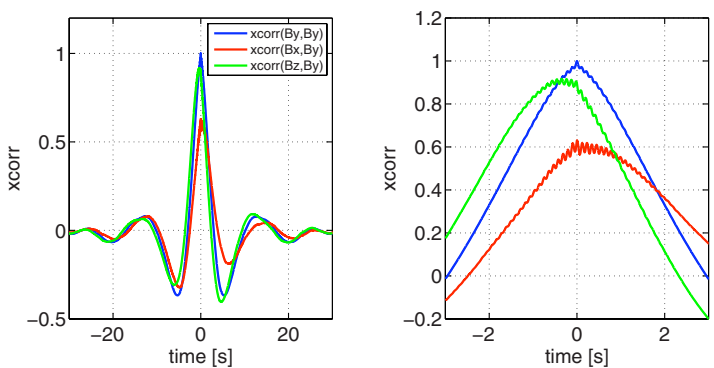

(b)
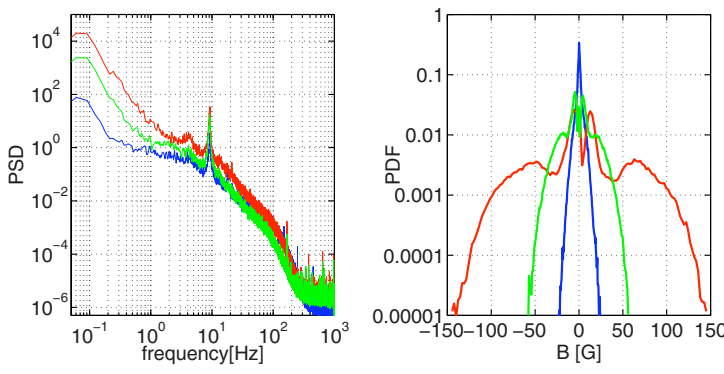

FIG. 26. (Color online) Bursting regimes, VKS2h; $F_{1}=21 \mathrm{~Hz}, F_{2}=15 \mathrm{~Hz}$, $\Theta=0.167$ [cf. Fig. 25]. (a) Time correlations of the magnetic field components. (b) Zoom around zero time lags. (c) Corresponding time-power spectra. (d) PDFs of the three magnetic field components. Color code for (c) and (d) as in Fig. 25. 


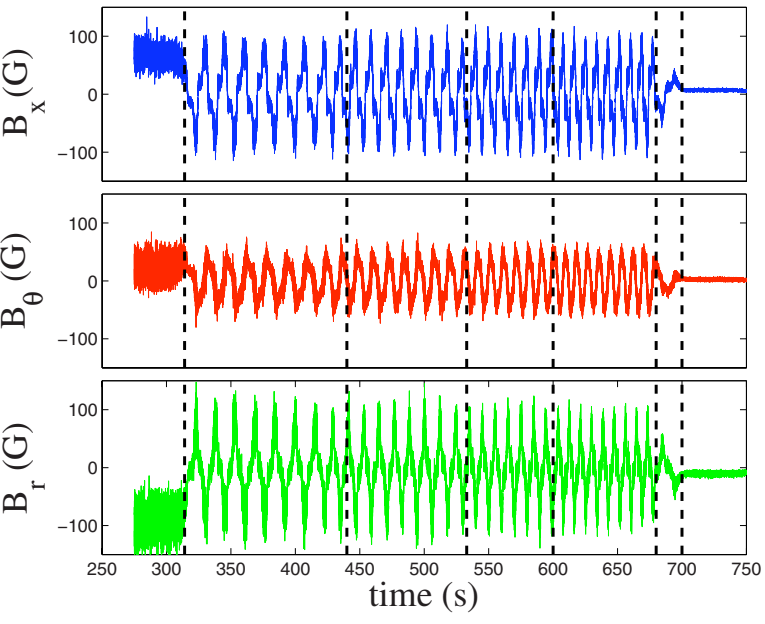

(a)
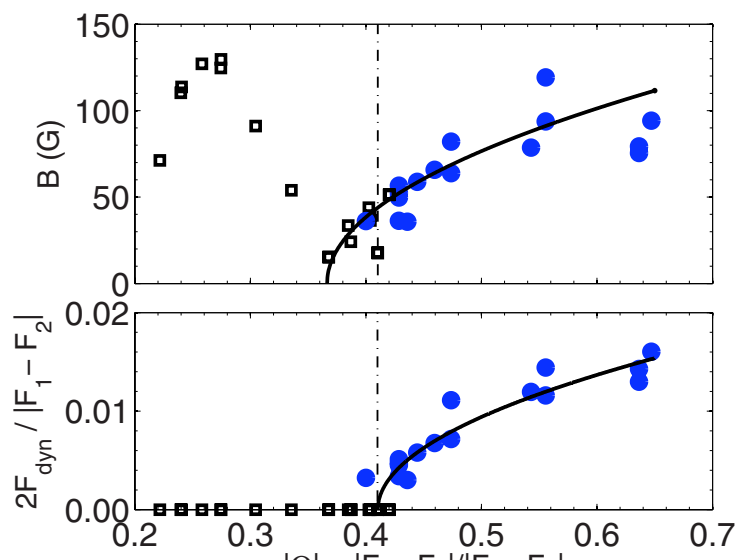

(b)

$|\Theta|=\left|F_{1}-F_{2}\right| /\left|F_{1}+F_{2}\right|$

FIG. 27. (Color online) (a) Oscillatory time signals for $\Theta$ in the range of $0.44-0.56$. The vertical dashed lines denote impeller frequency changes. Starting with $F_{1}=28 \mathrm{~Hz}$ kept constant, we observe successively: stationary dynamo $\left(F_{2}=12 \mathrm{~Hz} ;|\Theta|=0.40\right)$ and oscillatory dynamo $\left(F_{1}\right.$ $=11,10,9,8 \mathrm{~Hz} ; \Theta=0.44,0.47,0.51,0.56)$. Then $R_{m}$ is reduced below dynamo onset by lowering $F_{2}$ to $25 \mathrm{~Hz}$ (damped oscillations) and $20 \mathrm{~Hz}$ (no dynamo). (b): Evolution of the magnetic field amplitude and the dimensionless oscillation frequency with $\Theta$ for Osc oscillatory dynamo regimes (closed circles) and Stat3 stationary dynamo regimes (open squares). Amplitude of oscillations is a peak value (see text). Quadratic fits are described in text and the vertical line stands for the oscillatory mode threshold $\Theta_{o}$ $=0.41$.

\section{G. Oscillatory regimes}

Oscillatory dynamos are observed when $|\Theta|>0.4$. Figure 27(a) shows a temporal signal obtained when $\Theta$ is varied from 0.44 to 0.56 at constant $F_{1}=28 \mathrm{~Hz}$. The frequency of oscillation $F_{\text {dyn }}$ increases with $|\Theta|$. Following different paths in the $\left(R_{m}, \Theta\right)$ plane, we have observed that the oscillations are reached as a secondary instability of the Stat3 stationary dynamo. Although more systematic studies are needed, we suspect that the oscillatory behavior is path dependent [cf. Fig. 18(b)].

We study the bifurcation as a function of $\Theta$, while $R_{m}$ is kept in the range $\mathrm{e}^{31-36}$ [cf. Fig. 18(b)]. The evolution of the amplitude of the magnetic field with $|\Theta|$ is plotted in Fig. 27(b) for the Stat3 stationary regime and the oscillatory regime (Osc). One first observes that the amplitude of Stat3 is maximum around $|\Theta|=0.27$, reaches a minimum at $|\Theta|=\Theta_{b}$ $=0.37$, very close to zero, and starts increasing again. We also observe that the fluctuation level is high below $\Theta_{b}$ $=0.37$ [Fig. 19(i), $\Theta=-0.22$ ] and negligible above [see Fig. 19(j), $\Theta=-0.42]$. We conclude that we are in presence of two different stationary regimes (Stat3a and Stat3b exchanging their stability near $\pm \Theta_{b}$ ). The second regime Stat3b bifurcates toward oscillations at finite amplitude around $|\Theta|=0.4$. Above, the amplitude of the oscillations is computed as $B^{2}$ $=B_{x, \text { max }}^{2}+B_{y, \text { max }}^{2}+B_{z, \text { max }}^{2}$, where $B_{i, \text { max }}$ is the peak amplitude of the $i$-component. With this definition, we observe a continuity between the amplitude of Stat $3 \mathrm{~b}$ and the oscillation regime. A quadratic fit reveals a $B \propto\left(|\Theta|-\Theta_{b}\right)^{1 / 2}$ dependence and confirms $\Theta_{b}=0.37$.

The dimensionless oscillation frequency $\left(2 F_{\text {dyn }} / \mid F_{1}\right.$ $\left.-F_{2} \mid\right)$ is shown in Fig. 27(b). It behaves as $\left(|\Theta|-\Theta_{o}\right)^{1 / 2}$, confirming the oscillatory regime threshold value $\Theta_{o}=0.41$. This low frequency oscillatory dynamo mode appears as a finiteamplitude and zero-frequency secondary bifurcation as in low dimensional dynamical systems. Far from the onset, the almost-saturated frequency $F_{\text {dyn }}$ is of the order of 0.014 times the rotation frequency. The corresponding period is much larger than any hydrodynamical or magnetohydrodynamical characteristic time scales. It is two orders of magnitude larger than the period associated with the global rotation $\left|F_{1}-F_{2}\right| / 2$ or to the average frequency $F=\left(F_{1}+F_{2}\right) / 2$ and three orders of magnitude larger than the magnetic diffusion time.

\section{H. Transition and dynamics}

We have reported so far a great variety of different dynamical regimes in the parameter space $\left(F_{1}, F_{2}\right)$ : for impellers in counter-rotation at roughly equal frequencies, only statistically stationary dynamos have been observed. Oscillatory regimes exist when one impeller rotates much faster than the other, although a stationary dynamo has been also observed with only one rotating impeller. Experiments on hydrodynamical instabilities have shown since a long time that the competition between a stationary and an oscillatory instability leads to complex behaviors ${ }^{83-85}$ that can be understood in the framework of bifurcations of multiple codimension. ${ }^{86}$ Competing critical modes have also been considered as phenomenological models of the dynamics of the magnetic fields of the $\operatorname{Earth}^{87,88}$ or the $\operatorname{Sun}^{89,90}$ and the same analytical techniques have been used to describe the resulting dynamics. It has been also observed in simulations $^{91}$ or mean field models ${ }^{92}$ that magnetic field reversals can occur when a stationary and an oscillatory mode is in proximity and a simple analytic model has been designed in this spirit. ${ }^{93}$ However, it is far from obvious that these low dimensional dynamical features that have been observed in experiments involving laminar flows, analytical models, and numerical simulations at moderate kinetic Reynolds numbers can survive in strongly turbulent flows. One can, for instance, imagine that strong turbulent fluctuations smear them out by inducing transitions between different dynamo regimes corresponding to different realizations (in the statistical meaning) of the turbulent flow. We will show in this section that this is not the case. Trajectories plotted in low dimensional projections of the phase space are remark- 
(a)

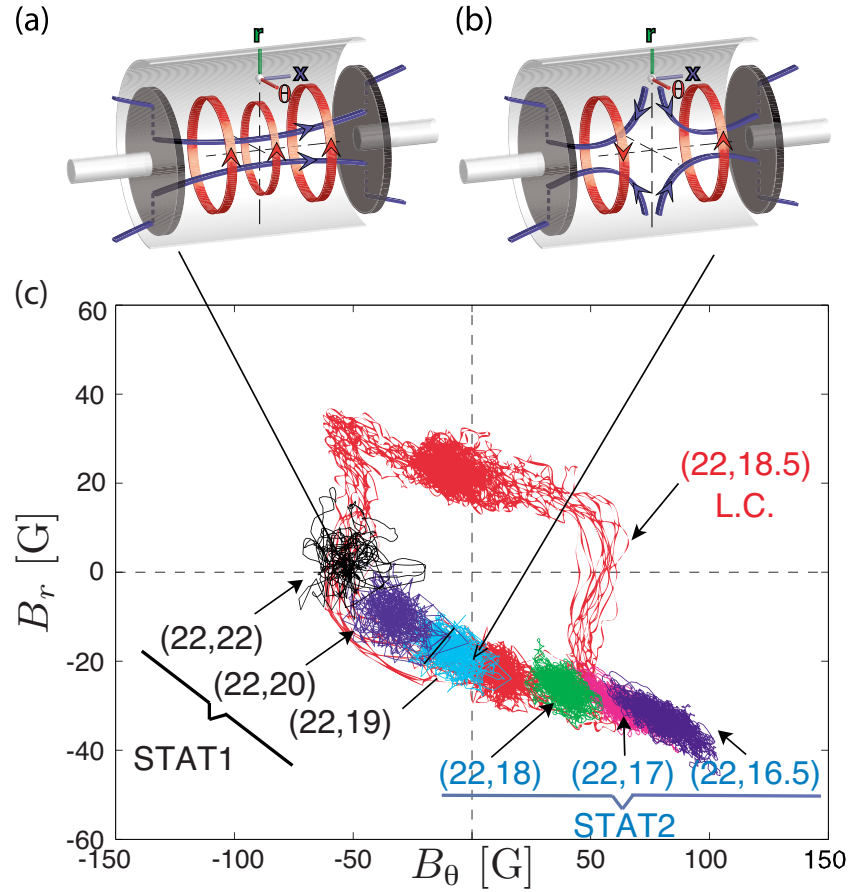

(d)

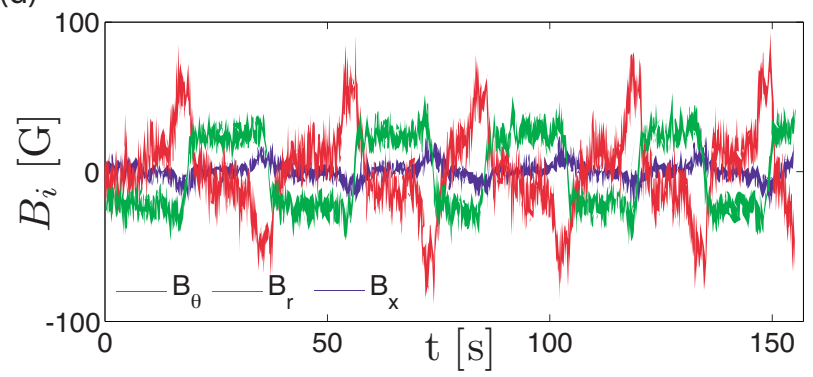

FIG. 28. (Color online) Sketch of the axial dipolar (a) and quadrupolar (b) magnetic modes. (c) Location of the different states in the $\left(B_{r}, B_{\theta}\right)$ plane: fixed points corresponding to the stationary regimes for frequencies $\left(F_{1}, F_{2}\right)$; limit cycle (LC) observed for impellers counter-rotating at different frequencies $(22,18.5) \mathrm{Hz}$ (red cycle). The magnetic field is time averaged over $1 \mathrm{~s}$ to remove high frequency fluctuations caused by the turbulent velocity fluctuations. (d) Time recording of the components of the magnetic field for frequencies $(22,18.5) \mathrm{Hz}(\Theta=0.086)$.

ably robust and bifurcations between different dynamo regimes do exhibit well known features of low dimensional dynamical systems. Complex dynamical regimes result from the existence of different stationary dynamo states that become unstable when $F_{1}$ or $F_{2}$ are varied within a small interval range.

We first consider how the Stat1 dynamo regime bifurcates to a time-dependent regime (LC) when the frequency of an impeller, say $F_{2}$, is decreased, while $F_{1}$ is kept constant, i.e., starting from $\Theta=0$ and increasing $|\Theta|$. One starts from a statistically stationary dynamo regime with a dominant azimuthal mean field close to the flow periphery [Fig. 28(a)]. This corresponds to the trace labeled $(22,22)$ in the $\left(B_{\theta}, B_{r}\right)$ plane of Fig. 28(c). As the frequency is decreased, the radial component of the mean field increases and then becomes larger than the azimuthal one $[(22,20)$ and $(22,19)]$. When we tune the impeller frequencies to $(22,18.5)$ $\mathrm{Hz}-\Theta=0.086-\mathrm{a}$ global bifurcation to a LC occurs [Figs. 28(c) and 28(d)]. We observe that the trajectory of this LC goes through the location of the previous fixed points related to the stationary regimes. This transition thus looks like the one of an excitable system: an elementary example of this type of bifurcation is provided by a simple pendulum submitted to a constant torque. As the value of the torque is increased, the stable equilibrium of the pendulum becomes more and more tilted from the vertical and for a critical torque corresponding to the angle $\pi / 2$, the pendulum undergoes a saddle-node bifurcation to a LC that goes through the previous fixed points. The magnetic field being measured at the periphery of the flow in the midplane between the two impellers, we observe that there is a strong radial component [green trace in Fig. 28(d)] that switches between \pm 25 G. We propose to ascribe it to a quadrupolar mode [see Fig. 28(b)]. Its interaction with the dipolar mode [Fig. 28(a)] that is the dominant one for exact counter-rotation gives rise to the observed relaxation dynamics. This hypothesis is supported by measurements made outside of the equatorial plane $(x$ $=109 \mathrm{~mm}, \quad r=206 \mathrm{~mm}$ ) where the radial to azimuthal field ratio is much smaller, as it should if the radial field mostly results from the quadrupolar component. We note that it has been often observed that dipolar and quadrupolar dynamo modes can have their respective thresholds in a narrow range of $R_{m}$ (Ref. 2) and this has been used to model the dynamics of the magnetic fields of the Earth ${ }^{87}$ or the Sun. ${ }^{90}$ The relaxation oscillation is observed in a rather narrow range of impeller frequency $F_{2}$ (less than $1 \mathrm{~Hz}$ ). When the frequency of the slowest impeller is decreased further, statistically stationary regimes are recovered $[(22,18)$ to $(22,16.5)$ in Fig. 28 (c)]. They also correspond to fixed points located on the trajectory of the LC, except for the case $(22,16.5) \mathrm{Hz}$ that separates from it $(\Theta=0.147)$.

When the rotation frequency of the slowest impeller is decreased further, new dynamical regimes occur. One of them consists in field reversals described in Sec. III D. The three components of the magnetic field reverse at random times [Fig. 21(a)]. Compared to the relaxation cycle described above, the length of phases with given polarity has a wider distribution. However, a variation in temperature of the sodium is enough to transform random reversals to nearly periodic noisy oscillations. Even in the case of random reversals, e.g., for the data in Fig. 21, if we plot the trajectories connecting $\mathbf{B}$ and $\mathbf{- B}$ in a phase space reconstruction, we obtain an average LC displayed by a thick dashed black line in Fig. 29. The state with positive polarity corresponds to point $\mathrm{P}$. When a reversals starts, the system leaves $\mathrm{P}$ and slows down in the vicinity of point $\mathrm{Q}$ that corresponds to the end of the exponential decay of the magnetic field recorded in Fig. 21(b). The trajectory then abruptly changes direction in phase space and leaves Q. This corresponds to the fast recovery of the magnetic field amplitude in Fig. 21(b). Finally, the trajectory reaches the point $-\mathrm{P}$ corresponding to the phase of negative polarity by circling around it, which corresponds to the overshoot in the direct time recording [Fig. 21(b)]. As proposed by many authors, ${ }^{88}$ it is tempting to understand these field reversals as heteroclinic orbits connecting unstable fixed points in phase space. In the present situation, these fixed points are P, Q together with the symmetric ones $-\mathrm{P}$ and $-\mathrm{Q}$ obtained by the $\mathbf{B} \rightarrow-\mathbf{B}$ transforma- 


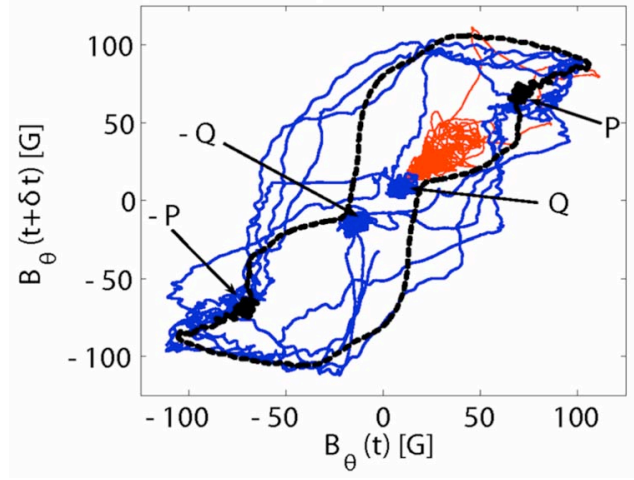

FIG. 29. (Color online) Plot of a cut in a phase space reconstruction $\left[B_{\theta}(t), B_{\theta}(t+\delta t)\right]$ with $\delta t=1 \mathrm{~s}$ for three regimes: thick dashed black line for field reversals reported in Fig. 21, the magnetic field being rescaled by an $a d$ hoc factor accounting for the fact that the probe location is not in the midplane. The medium blue line is for symmetric bursts $\left(F_{1}=21 \mathrm{~Hz}, F_{2}\right.$ $=15 \mathrm{~Hz}, \Theta=0.17)$ and the thin red line for asymmetric bursts $\left(F_{1}\right.$ $\left.=22 \mathrm{~Hz}, F_{2}=15 \mathrm{~Hz}, \Theta=0.19\right)$. In these last two plots the magnetic field is time averaged over $0.25 \mathrm{~s}$ to remove high frequency fluctuations.

tion. The trajectory in phase space is amazingly robust despite strong velocity fluctuations. These fluctuations set an upper bound on the duration of phases with a given polarity by preventing the system from staying too close to the unstable fixed points and thus to spend too much time in their vicinity. However, this does not suppress the scale separation between the length of the phases with given polarity and the duration of a reversal. In that sense, turbulent fluctuations have a weak effect on the large scale dynamics of the magnetic field. Furthermore, as mentioned in Sec. III D, reversals observed in almost identical conditions in the VKS2h experiments follow a slightly different path in the phase space: at each polarity change a different path in phase space is selected which does not systematically get very close to the unstable fixed points $\mathrm{Q}$ and $-\mathrm{Q}$.

We will show next that the fixed points $\pm \mathrm{P}$ and $\pm \mathrm{Q}$ are also involved in other dynamical regimes observed in the vicinity of reversals in the parameter space and shown in Fig. 29. For rotation frequencies $(22,15) \mathrm{Hz}-|\Theta|=0.19$ - the magnetic field displays intermittent bursts in which the most probable value of the azimuthal field is roughly $20 \mathrm{G}$ but bursts up to more than $100 \mathrm{G}$ are observed [see Sec. III E, Fig. 23(a)]. For rotation frequencies $(21,15) \mathrm{Hz}-|\Theta|=0.17$ - the same type of dynamics occur, but in a symmetric fashion, both positive and negative values of the field being observed [Sec. III E, Fig. 25(a)]. A section of a phase space reconstruction is displayed in Fig. 29 for both regimes of bursts and field reversals. We observe that the asymmetric bursts occur from the unstable fixed point $Q$ in the vicinity of which the signal is located most of the time, whereas symmetric burst involve the two unstable fixed points $\pm \mathrm{Q}$. Therefore, these complex dynamics, as well as reversals, can be understood as resulting from the competition between different dynamo modes corresponding to the fixed points $\pm \mathrm{P}$ and $\pm \mathrm{Q}$ that bifurcate from stable to unstable in a small region of parameter space.

The different dynamical regimes of the magnetic field generated by the VKS flow thus display several characteristic features of low dimensional dynamical systems, besides fixed points and weakly anharmonic oscillations: global bifurcations from fixed points to a relaxation cycle (Fig. 28), chaotic bursts from unstable fixed points (Fig. 29). These dynamics can be understood as the ones resulting from the competition between a few nearly critical modes.

\section{CONCLUDING REMARKS}

The experimental observations reported here make a first step toward a detail investigation of dynamo action and its dynamical regimes in laboratory experiments. This is an essential step between numerical simulations, still far from the realm of realistic fluid dynamos, and planetary and stellar dynamos.

The advantage of the von Kármán geometry investigated here is that fundamental features such as turbulence and global rotation are incorporated. As detailed here, the VKS experiment has brought the following new results:

(a) When the flow is driven with exact counter-rotation, the observed dynamo cannot be explained by the action of the mean flow alone. Turbulence is essential. This is different from previous experiments for which the dynamo's essential features could be predicted by taking into account only the time-averaged flow.

(b) When global rotation is added, a small variation in parameters has allowed for the first time the observation of rich dynamical regimes. Some of these, like intermittent reversals or regular oscillations, are strikingly similar to the behavior of the geomagnetic and solar magnetic fields. A noteworthy feature is that the geometry and time dynamics of these regimes are robust with respect to the turbulent fluctuations, known to be large inside the flow. The turbulent fluctuations do not induce intermittent transitions between magnetic regimes even though they lie in closely adjacent or even overlapping parameter ranges. ${ }^{79}$

There are nevertheless still many open and intriguing questions left from the preliminary observations reported here. To quote but a few:

(a) The effect of the soft-iron impellers. They do change profoundly the self-generation capacity of the flow since identical runs with stainless steel impellers have not self-generated in the same range of flow driving parameters. They may also select a particular type of dynamo. Induction measurements below threshold are indeed quite different from previous VKS observations with stainless steel drives. We finally note that, in two particular runs-at the first dynamo run and after some oscillatory dynamo regime-for which the setup magnetization was probably very low, regimes without dynamo action at $\Theta=0$ were observed above onset between $R_{m}=31(F=16 \mathrm{~Hz})$ and $R_{m}=39(F=20 \mathrm{~Hz})$.

(b) The dynamics of the Lorentz force during the nonlinear saturation. The way in which it modifies the velocity field when a $(\mathbf{u}, \mathbf{B} \neq 0)$ state is reached must yet be 
studied, as well as how it acts in the dynamical regimes.

(c) Also open is the interaction between the hydrodynamics and the magnetic field modes. The von Kármán flow displays slow time dynamics and transitions between different flow regimes.

Finally, the question of dynamo generation in a fully homogeneous container is still an open question. Is the present VKS dynamo a nearly homogeneous dynamo? If the presence of soft-iron just reduces the dynamo threshold into the accessible experimental range without major modification of the nonlinear dynamics, the answer is clearly yes. However, if a coupling responsible for dynamo generation and characteristics, such as mode selection and dynamics, explicitly involves the distribution of ferromagnetic elements, then the VKS2 dynamo will have to be considered as an inhomogeneous one. Future experimental investigations in the VKS experiments are aimed at identifying the essential ingredients for dynamo generation in von Kármán flowsincluding the role of electrical and magnetic boundary conditions - and the implication of these elements on mode selection and dynamical properties.

\section{ACKNOWLEDGMENTS}

We thank M. Moulin, C. Gasquet, J.-B Luciani, A. Skiara, D. Courtiade, J.-F. Point, P. Metz, and V. Padilla for their technical assistance. This work was supported by Grant No. ANR05-0268-03, Direction des Sciences de la Matière et Direction de l'Énergie Nucléaire of CEA, Ministère de la Recherche and CNRS. The experiment was operated at CEA/ Cadarache DEN/DTN.

${ }^{1}$ J. Larmor, "How could a rotating body such as the Sun become a magnet?" Br. Assoc. Adv. Sci., Rep., 159 (1919).

${ }^{2}$ H. K. Moffatt, Magnetic Field Generation in Electrically Conducting Fluids (Cambridge University Press, Cambridge, 1978).

${ }^{3}$ F. J. Lowes and I. Wilkinson, "Geomagnetic dynamo: A laboratory model," Nature (London) 198, 1158 (1963).

${ }^{4}$ F. J. Lowes and I. Wilkinson, "Geomagnetic dynamo: An improved laboratory model," Nature (London) 219, 717 (1968).

${ }_{5}^{5}$ A. Gailitis, O. Lielausis, E. Platacis, S. Dementev, A. Cifersons, G. Gerbeth, T. Gundrum, F. Stefani, M. Christen, and G. Will, "Magnetic field saturation in the Riga dynamo experiment," Phys. Rev. Lett. 86, 3024 (2001).

${ }^{6}$ Yu. B. Ponomarenko, "Theory of the hydromagnetic generator," J. Appl. Mech. Tech. Phys. 14, 775 (1973).

${ }^{7}$ A. Gailitis, O. Lielausis, E. Platacis, G. Gerbeth, and F. Stefani, "The Riga dynamo experiment," Surv. Geophys. 24, 247 (2003).

${ }^{8}$ S. Kenjeres and K. Hanjalic, "Numerical simulation of a turbulent magnetic dynamo," Phys. Rev. Lett. 98, 104501 (2007).

${ }^{9}$ S. Kenjeres and K. Hanjalic, "Numerical insights into magnetic dynamo action in turbulent regime," New J. Phys. 9, 306 (2007).

${ }^{10}$ R. Stieglitz and U. Müller, "Experimental demonstration of a homogeneous two-scale dynamo," Phys. Fluids 13, 561 (2001).

${ }^{11}$ G. O. Roberts, "Dynamo action of fluid motions with two-dimensional periodicity," Philos. Trans. R. Soc. London, Ser. A 271, 411 (1972).

${ }^{12}$ A. Gilbert and P.-L. Sulem, "On inverse cascade in alpha-effect dynamos," Geophys. Astrophys. Fluid Dyn. 51, 243 (1990).

${ }^{13}$ A. Tilgner, "A kinematic dynamo with a small scale velocity field," Phys. Lett. A 226, 75 (1997).

${ }^{14}$ A. Tilgner and F. H. Busse, "Saturation mechanism in a model of Karlsruhe dynamo," in Dynamo and Dynamics: A Mathematical Challenge, edited by P. Chossat, D. Armbruster, and I. Oprea (Kluwer, Dordrecht, 2001), pp. 109-116.
${ }^{15}$ K.-H. Rädler, E. Apstein, M. Rheinhardt, and M. Schüler, "The Karlsruhe dynamo experiment. A mean field approach," Stud. Geophys. Geod. 42, 224 (1998).

${ }^{16}$ R. Monchaux, M. Berhanu, M. Bourgoin, M. Moulin, Ph. Odier, J.-F. Pinton, R. Volk, S. Fauve, N. Mordant, F. Pétrélis, A. Chiffaudel, F. Daviaud, B. Dubrulle, C. Gasquet, L. Marié, and F. Ravelet, "Generation of magnetic field by dynamo action in a turbulent flow of liquid sodium," Phys. Rev. Lett. 98, 044502 (2007).

${ }^{17}$ M. Berhanu, R. Monchaux, M. Bourgoin, M. Moulin, Ph. Odier, J.-F. Pinton, R. Volk, S. Fauve, N. Mordant, F. Pétrélis, A. Chiffaudel, F. Daviaud, B. Dubrulle, C. Gasquet, L. Marié, and F. Ravelet, "Magnetic field reversals in an experimental turbulent dynamo," Europhys. Lett. 77, 59001 (2007).

${ }^{18}$ F. Ravelet, R. Monchaux, M. Berhanu, S. Aumaître, A. Chiffaudel, F. Daviaud, B. Dubrulle, M. Bourgoin, Ph. Odier, J.-F. Pinton, N. Plihon, R. Volk, S. Fauve, N. Mordant, and F. Pétrélis, "Chaotic dynamos generated by a turbulent flow of liquid sodium," Phys. Rev. Lett. 101, 074502 (2008).

${ }^{19}$ M. Bourgoin, L. Marié, F. Pétrélis, C. Gasquet, A. Guigon, J.-B. Luciani, M. Moulin, F. Namer, J. Burguete, A. Chiffaudel, F. Daviaud, S. Fauve, $\mathrm{Ph}$. Odier, and J.-F. Pinton, "Magnetohydrodynamics measurements in the von Kármán sodium experiment," Phys. Fluids 14, 3046 (2002).

${ }^{20}$ F. Pétrélis, M. Bourgoin, L. Marié, J. Burguete, A. Chiffaudel, F. Daviaud, $\mathrm{S}$. Fauve, Ph. Odier, and J.-F. Pinton, "Nonlinear magnetic induction by helical motion in a liquid sodium turbulent flow," Phys. Rev. Lett. 90, 174501 (2003).

${ }^{21} \mathrm{~L}$. Marié, "Transport de moment cinétique et de champ magnétique par un écoulement tourbillonnaire turbulent: Influence de la rotation," Ph.D. thesis, Université de Paris 7, 2003 (http://tel.archives-ouvertes.fr/tel00007755/en/).

${ }^{22}$ F. Ravelet, "Bifurcations globales hydrodynamiques et magnétohydrodynamiques dans un écoulement de von Kármán turbulent," Ph.D. thesis, École Polytechnique, 2005 (http://tel.archives-ouvertes.fr/tel-00011016/ en/).

${ }^{23} \mathrm{R}$. Monchaux, "Mécanique statistique et effet dynamo dans un écoulement de von Kármán turbulent," Ph.D. thesis, Université Diderot, Paris 7, 2007 (http://tel.archives-ouvertes.fr/tel-00199751/en/).

${ }^{24}$ S. Douady, Y. Couder, and M.-E. Brachet, "Direct observation of the intermittency of intense vorticity filaments in turbulence," Phys. Rev. Lett. 67, 983 (1991).

${ }^{25}$ S. Fauve, C. Laroche, and B. Castaing, "Pressure fluctuations in swirling turbulent flows," J. Phys. II 3, 271 (1993).

${ }^{26}$ J.-F. Pinton and R. Labbé, "Correction to Taylor hypothesis in swirling flows," J. Phys. II 4, 1461 (1994).

${ }^{27}$ J. Maurer, P. Tabeling, and G. Zocchi, "Statistics of turbulence between two counter rotating disks in low temperature helium gas," Europhys. Lett. 26, 31 (1994).

${ }^{28}$ R. Labbé, J.-F. Pinton, and S. Fauve, "Power fluctuations in turbulent swirling flows," J. Phys. II 6, 1099 (1996).

${ }^{29}$ L. Marié and F. Daviaud, "Experimental measurement of the scale-byscale momentum transport budget in a turbulent shear flow," Phys. Fluids 16, 457 (2004).

${ }^{30}$ F. Ravelet, A. Chiffaudel, and F. Daviaud, "Supercritical transition to turbulence in an inertially driven von Kármán closed flow," J. Fluid Mech. 601, 339 (2008).

${ }^{31}$ P.-P. Cortet, P. Diribarne, R. Monchaux, A. Chiffaudel, F. Daviaud, and B. Dubrulle, "Normalized kinetic energy as a hydrodynamical global quantity for inhomogeneous anisotropic turbulence," Phys. Fluids 21, 025104 (2009).

${ }^{32}$ M. L. Dudley and R. W. James, "Time-dependent kinematic dynamos with stationary flows," Proc. R. Soc. London, Ser. A 425, 407 (1989).

${ }^{33}$ L. Marié, C. Normand, and F. Daviaud, "Galerkin analysis of kinematic dynamos in the von Kármán geometry," Phys. Fluids 18, 017102 (2006).

${ }^{34}$ L. Marié, J. Burguete, F. Daviaud, and J. Léorat, "Numerical study of homogeneous dynamo based on experimental von Kármán type flows," Eur. Phys. J. B 33, 469 (2003).

${ }^{35}$ F. Ravelet, A. Chiffaudel, F. Daviaud, and J. Léorat, "Toward an experimental von Kármán dynamo: Numerical studies for an optimized design," Phys. Fluids 17, 117104 (2005).

${ }^{36}$ M. Bourgoin, P. Odier, J.-F. Pinton, and Y. Ricard, "An iterative study of time independent induction effects in magnetohydrodynamics," Phys. Fluids 16, 2529 (2004).

${ }^{37}$ M. Bourgoin, 'Etudes en magnétohydrodynamique, application á l'effet 
dynamo," Ph.D. thesis, Ecole Normale Supérieure de Lyon, 2003 (http:// tel.archives-ouvertes.fr/tel-00008302/en/).

${ }^{38}$ F. Stefani, M. Xu, G. Gerbeth, F. Ravelet, A. Chiffaudel, F. Daviaud, and J. Léorat, "Ambivalent effects of added layers on steady kinematic dynamos in cylindrical geometry: Application to the VKS experiment," Eur. J. Mech. B/Fluids 25, 894 (2006).

${ }^{39} \mathrm{~S}$. Fauve and F. Pétrélis, in The Dynamo Effect, Peyresq Lectures on Nonlinear Phenomena Vol. II, edited by J.-A. Sepulchre (World Scientific, Singapore, 2003), pp. 1-64.

${ }^{40}$ F. Pétrélis, "Effet dynamo: Etudes des mécanismes d'instabilité et de saturation du champ magnétique," Ph.D. thesis, Université Pierre et Marie Curie-Paris VI, 2002 (http://tel.archives-ouvertes.fr/tel-00003842/en/).

${ }^{41} \mathrm{~F}$. Pétrélis and S. Fauve, "Inhibition of the dynamo effect by phase fluctuations," Europhys. Lett. 76, 602 (2006).

${ }^{42} \mathrm{M}$. Brachet, "Direct simulation of three-dimensional turbulence in the Tayor-Green vortex," Fluid Dyn. Res. 8, 1 (1991).

${ }^{43}$ C. Nore, M. Brachet, H. Politano, and A. Pouquet, "Dynamo action in the Taylor-Green vortex near threshold," Phys. Plasmas 4, 1 (1997).

${ }^{44}$ Y. Ponty, H. Politano, A. Pouquet, and J.-F. Pinton, "Simulation of induction at low magnetic Prandtl number," Phys. Rev. Lett. 92, 144503 (2004).

${ }^{45}$ Y. Ponty, P. D. Minnini, D. Montgomery, J.-F. Pinton, H. Politano, and A. Pouquet, "Numerical study of dynamo action at low magnetic Prandtl numbers," Phys. Rev. Lett. 94, 164502 (2005).

${ }^{46}$ J.-P. Laval, P. Blaineau, N. Leprovost, B. Dubrulle, and F. Daviaud, "Influence of turbulence on the dynamo threshold," Phys. Rev. Lett. 96, 204503 (2006)

${ }^{47}$ B. Dubrulle, P. Blaineau, O. Mafra Lopes, F. Daviaud, J.-P. Laval, and R. Dolganov, "Bifurcations and dynamo action in a Taylor-Green flow," New J. Phys. 9, 308 (2007).

${ }^{48}$ A. A. Schekochihin, S. Cowley, G. Hammett, J. Maron, and J. C. McWilliams, "A model of nonlinear evolution and saturation of the turbulent MHD dynamo," New J. Phys. 4, 84 (2002).

${ }^{49}$ A. A. Schekochihin, S. Cowley, J. Maron, and J. C. McWilliams, "Critical magnetic Prandtl number for small-scale dynamo," Phys. Rev. Lett. 92, 054502 (2004)

${ }^{50}$ A. B. Iskakov, A. A. Schekochihin, S. C. Cowley, J. C. McWilliams, and M. R. E. Proctor, "Numerical demonstration of fluctuation dynamo at low magnetic Prandtl numbers," Phys. Rev. Lett. 98, 208501 (2007).

${ }^{51}$ D. P. Lathrop, W. L. Shew, and D. R. Sisan, "Laboratory experiments on the transition to MHD dynamos," Plasma Phys. Controlled Fusion 43, A151 (2001).

${ }^{52}$ N. L. Peffley, A. G. Goumilevski, A. B. Cawthrone, and D. P. Lathrop, "Characterization of experimental dynamos," Geophys. J. Int. 142, 52 (2000).

${ }^{53}$ N. L. Peffley, A. B. Cawthorne, and D. P. Lathrop, "Toward a selfgenerating magnetic dynamo: The role of turbulence," Phys. Rev. E 61, 5287 (2000)

${ }^{54}$ E. J. Spence, M. D. Nornberg, C. M. Jacobson, C. A. Parada, N. Z. Taylor, R. D. Kendrick, and C. B. Forest, "Turbulent diamagnetism in flowing liquid sodium," Phys. Rev. Lett. 98, 164503 (2007).

${ }^{55}$ M. D. Nornberg, E. J. Spence, R. D. Kendrick, C. M. Jacobson, and C. B. Forest, "Intermittent magnetic field excitation by a turbulent flow of liquid sodium," Phys. Rev. Lett. 97, 044503 (2006).

${ }^{56}$ M. D. Nornberg, E. J. Spence, R. D. Kendrick, C. M. Jacobson, and C. B. Forest, "Measurements of the magnetic field induced by a turbulent flow of liquid metal," Phys. Plasmas 13, 055901 (2006).

${ }^{57}$ D. Sweet, E. Ott, J. M. Finn, T. M. Antonsen, and D. P. Lathrop, "Blowout bifurcations and the onset of magnetic activity in turbulent dynamos," Phys. Rev. E 63, 066211 (2001).

${ }^{58}$ D. Sweet, E. Ott, T. M. Antonsen, and D. P. Lathrop, "Blowout bifurcations and the onset of magnetic dynamo action," Phys. Plasmas 8, 1944 (2001).

${ }^{59}$ M. Bourgoin, R. Volk, N. Plihon, P. Augier, and J.-F. Pinton, "A Bullard von Kármán dynamo," New J. Phys. 8, 329 (2006).

${ }^{60}$ R. Avalos-Zuniga, F. Plunian, and A. Gailitis, "Influence of electromagnetic boundary conditions onto the onset of dynamo action in laboratory experiments," Phys. Rev. E 68, 066307 (2003).

${ }^{61}$ C. Gissinger, A. Iskakov, S. Fauve, and E. Dormy, "Effect of magnetic boundary conditions on the dynamo threshold of von Kármán swirling flows," Europhys. Lett. 82, 29001 (2008).

${ }^{62}$ N. Mordant, J.-F. Pinton, and F. Chillà, "Characterization of turbulence in a closed flow," J. Phys. II 7, 1729 (1997).

${ }^{63}$ R. Volk, R. Monchaux, M. Berhanu, F. Ravelet, A. Chiffaudel, F. Daviaud,
B. Dubrulle, S. Fauve, N. Mordant, Ph. Odier, F. Pétrélis, and J.-F. Pinton, "Transport of magnetic field by a turbulent flow of liquid sodium," Phys. Rev. Lett. 97, 074501 (2006).

${ }^{64}$ F. Pétrélis, N. Mordant, and S. Fauve, "On the magnetic fields generated by experimental dynamos," Geophys. Astrophys. Fluid Dyn. 101, 289 (2007).

${ }^{65}$ P. Odier, J.-F. Pinton, and S. Fauve, "Advection of a magnetic field by a turbulent swirling flow,” Phys. Rev. E 58, 7397 (1998).

${ }^{66}$ S. I. Braginsky, "Kinematic models of the Earth's hydromagnetic dynamo," Geomagn. Aeron. 4, 572 (1964).

${ }^{67}$ E. N. Parker, "Hydromagnetic dynamo models," Astrophys. J. 122, 293 (1955).

${ }^{68}$ F. Plunian and K.-H. Rädler, "Subharmonic dynamo action in the Roberts flow," Geophys. Astrophys. Fluid Dyn. 96, 115 (2002).

${ }^{69}$ R. Volk, P. Odier, and J.-F. Pinton, "Magnetic induction in a cylindrical array of helical vortices," Phys. Fluids 20, 016601 (2008).

${ }^{70}$ R. Laguerre, C. Nore, A. Ribeiro, J. Léorat, J.-L. Guermond, and F. Plunian, "Impact of impellers on the axisymmetric magnetic mode in the VKS2 dynamo experiment," Phys. Rev. Lett. 101, 104501 (2008).

${ }^{71}$ F. Krause and K. H. Radler, Mean-Field Magnetohydrodynamics and Dynamo Theory (Pergamon, Oxford, 1980).

${ }^{72}$ R. Stepanov, R. Volk, S. Denisov, P. Frick, V. Noskov, and J.-F. Pinton, "Induction, helicity and alpha effect in a toroidal screw flow of liquid gallium," Phys. Rev. E 73, 046310 (2006).

${ }^{73}$ R. Volk, "Fluctuations d'induction en magnétohydrodynamique, contributions à l'induction à grande échelle, application à l'effet dynamo," Ph.D. thesis, Ecole Normale Supérieure de Lyon, 2005 (http://tel.archivesouvertes.fr/tel-00011221/en/).

${ }^{74}$ K.-H. Rädler and R. Stepanov, "Mean electromotive force due to turbulence of a conducting fluid in the presence of mean flow," Phys. Rev. E 73, 056311 (2006).

${ }^{75}$ F. Pétrélis and S. Fauve, "Saturation of the magnetic field above the dynamo threshold," Eur. Phys. J. B 22, 273 (2001).

${ }^{76} \mathrm{~S}$. Fauve and F. Pétrélis, "Scaling laws of turbulent dynamos," C. R. Phys. 8, 87 (2007)

${ }^{77}$ P. D. Mininni, Y. Ponty, D. Montgomery, J.-F. Pinton, H. Politano, and A. Pouquet, "Nonlinear behavior of a non-helical dynamo," Astrophys. J. 626, 853 (2005).

${ }^{78}$ F. Ravelet, L. Marié, A. Chiffaudel, and F. Daviaud, "Multistability and memory effect in a highly turbulent flow: Experimental evidence for a global bifurcation," Phys. Rev. Lett. 93, 164501 (2004).

${ }^{79}$ M. Berhanu, B. Gallet, R. Monchaux, M. Bourgoin, Ph. Odier, J.-F. Pinton, N. Plihon, R. Volk, S. Fauve, N. Mordant, F. Pétrélis1, S. Aumaître, A. Chiffaudel, F. Daviaud, B. Dubrulle, and F. Ravelet, "Bistability between a stationary and an oscillatory dynamo in a turbulent flow of liquid sodium," J. Fluid Mech. (to be published).

${ }^{80}$ E. Dormy, J.-P. Valet and V. Courtillot, "Numerical models of the geodynamo and observational constraints," Geochem., Geophys., Geosyst. 1, 62 (2000).

${ }^{81}$ J.-P. Valet, "Time variations in geomagnetic intensity," Rev. Geophys. 41, 1004 (2003)

${ }^{82}$ J.-P. Valet, L. Meynadier, and Y. Guyodo, "Geomagnetic dipole strength and reversal rate over the past two million years," Nature (London) $\mathbf{4 3 5}$, 802 (2005).

${ }^{83}$ S. Fauve, C. Laroche, and B. Perrin, "Competing instabilities in a rotating layer of mercury heated from below," Phys. Rev. Lett. 55, 208 (1985).

${ }^{84}$ R. W. Walden, P. Kolodner, A. Passner, and C. M. Surko, "Traveling waves and chaos in convection in binary fluid mixtures," Phys. Rev. Lett. $\mathbf{5 5 ,} 496$ (1985).

${ }^{85}$ I. Rehberg and G. Ahlers, "Experimental observation of a codimension-2 bifurcation in a binary fluid mixture," Phys. Rev. Lett. 55, 500 (1985).

${ }^{86} \mathrm{~V}$. Arnold, Geometrical Methods in the Theory of Ordinary Differential Equations (Springer, Berlin, 1982).

${ }^{87}$ I. Melbourne, M. R. E. Proctor, and A. M. Rucklidge, in Dynamo and Dynamics: A Mathematical Challenge, edited by P. Chossat, D. Armbruster, and I. Oprea (Kluwer, Dordrecht, 2001), pp. 363-370.

${ }^{88} \mathrm{P}$. Chossat and D. Armbruster, "Dynamics of polar reversals in spherical dynamos," Proc. R. Soc. London, Ser. A 459, 577 (2003), and references therein.

${ }^{89}$ S. M. Tobias, N. O. Weiss, and V. Kirk, "Chaotically modulated stellar dynamos," Mon. Not. R. Astron. Soc. 273, 1150 (1995).

${ }^{90}$ E. Knobloch and A. S. Landsberg, "A new model of the solar cycle," Mon. Not. R. Astron. Soc. 278, 294 (1996). 
${ }^{91}$ G. R. Sarson and C. A. Jones, "A convection driven geodynamo reversal model," Phys. Earth Planet. Inter. 111, 3 (1999).

${ }^{92}$ F. Stefani and G. Gerbeth, "Asymmetric polarity reversals, bimodal field distribution, and coherence resonance in a spherically symmetric meanfield dynamo model," Phys. Rev. Lett. 94, 184506 (2005).

${ }^{93}$ P. Hoyng and J. J. Duistermaat, "Geomagnetic reversals and the stochastic exit problem," Europhys. Lett. 68, 177 (2004).
${ }^{94}$ R. Volk, P. Odier, and J.-F. Pinton, "Fluctuation of magnetic induction in von Kármán swirling flows," Phys. Fluids 18, 085105 (2006).

${ }^{95}$ R. Monchaux, F. Ravelet, B. Dubrulle, A. Chiffaudel, and F. Daviaud, "Properties of steady states in turbulent axisymmetric flows," Phys. Rev. Lett. 96, 124502 (2006).

${ }^{96} \mathrm{~A}$. de la Torre and J. Burguete, "Slow dynamics in a turbulent von Kármán swirling flow," Phys. Rev. Lett. 99, 054101 (2007). 